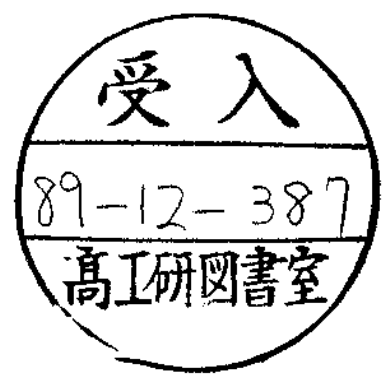

CERN-EP/89-112

4 September 1989

\title{
COMPARISON OF GLASS AND PLASTIC SCINTILLATING MICROFIBRES FOR HIGH-RESOLUTION TRACKING
}

\section{WA84 Collaboration}

C. Angelini ${ }^{4}$, W. Beusch ${ }^{2}$, A. Cardini ${ }^{4}$, D.J. Crennell ${ }^{1}$, M. De Vincenzi ${ }^{5}$, G. Di Vita ${ }^{5}$, A. Duane ${ }^{3}$, J.-P. Fabre ${ }^{2}$, V. Flaminio ${ }^{4}$, A. Frenkel ${ }^{5}$, T. Gys ${ }^{2}$, K. Harrison ${ }^{3}$, E. Lamanna ${ }^{5}$, H. Leutz ${ }^{2}$, D. Lucchesi ${ }^{4}$, G. Martellotti ${ }^{5}$, J.G. McEwen ${ }^{6}$, D.R.O. Morrison ${ }^{2}$, G. Penso ${ }^{5}$, S. Petrera ${ }^{5}$, C. Roda ${ }^{4}$, A. Sciubba ${ }^{5}$, E. Vicari ${ }^{2}$ and D.M. Websdale ${ }^{3}$

\begin{abstract}
The WA84 experiment at CERN has been proposed as a study of heavy-flavour physics using a novel, high-resolution vertex detector, consisting of an active target assembled from coherent bundles of scintillating optical microfibres. This paper is a presentation of work carried out in the development of the vertex detector. Results from tests of prototypes of the detector in a chargedparticle beam are reported, and the differences in the performance of glass and plastic active targets are discussed.
\end{abstract}

(Submitted to Nuclear Instruments and Methods in Physics Research)

1) Rutherford Appleton Laboratory, Didcot, UK.

2) CERN, Geneva, Switzerland.

3) Imperial College, London, UK.

4) Università di Pisa and INFN, Pisa, Italy.

5) Università di Roma 'La Sapienza' and INFN, Rome, Italy.

6) University of Southampton, Southampton, UK. 


\section{INTRODUCTION}

Particle detection using coherent bundles of scintillating fibres was originally proposed in the nineteen-fifties [1]. Development of the technique soon ceased, however, when it became clear that, with the technology of the time, the tracking capabilities of bubble chambers and of spark chambers were far superior. Contemporary high-energy particle physics experiments require precise tracking devices which are versatile, compact, and radiation-hard, and which have a fast response. A recent renewal of interest in the potential of scintillating-fibre detectors to fulfil these requirements has been stimulated by advances in optoelectronic technology.

The WA84 Collaboration at CERN is developing a scintillating-fibre active target for a study of the production and decay of beauty-flavoured hadrons [2]. This vertex detector is to be used in conjunction with the Omega Prime spectrometer [3] and a high intensity ( $\geqslant 10^{6}$ particles per second) $350 \mathrm{GeV} / \mathrm{c} \pi^{-}$beam. It is to provide event visualization in a plane that is transverse to the beam direction. A silicon microstrip telescope placed downstream from the target will aid in the matching of tracks seen in the target with those measured in the spectrometer.

The first of the targets tested were constructed from scintillating glass microfibres (diameter $\leqslant$ $30 \mu \mathrm{m}$ ), which had been shown to be highly resistant to radiation damage [4]. At the time when the WA84 experiment was proposed, the assembling of such fibres into targets having good coherence had already been successfully accomplished. The individual fibres were coated with a very thin layer of opaque material, referred to as an extra-mural absorber (EMA), which effectively restricted the passage of scintillation light from one fibre into another. Measurements by the WA84 Collaboration have demonstrated that in addition to the fast component reported elsewhere [4,5], for the glass fibres tested there are significant contributions from long-lived components of the light emission [6]. These contributions seriously limit the interaction rates that can be sustained.

Using plastic rather than glass scintillators has advantages that stem from the higher intrinsic light yield for the same energy deposition, from a shorter emission time, and from a lower atomic number. This had long been recognized, although initially there were two significant problems. First, for scintillating fibres to be useful it is necessary that the light emission take place within a distance from the point of energy deposition which is small compared with the fibre diameter, but in scintillating plastic microfibres in which this was possible the light transmission efficiency was unacceptably low. Secondly, there existed no EMA that was compatible with the fibre manufacturing process. The first of these problems has lately been overcome $[7,8]$, so that it has now been possible for targets constructed from scintillating plastic microfibres to be tested. Moreover, the newly developed plastic scintillators are sufficiently radiation-hard to be used in a high intensity particle beam, with negligible deterioration in performance for doses of up to $10^{3} \mathrm{~Gy}$.

This paper is a presentation of results from tests performed with cerium-doped GS1 glass fibres*) and with SCSN-81T(0.50) ${ }^{* *}$ and PMP-doped plastic fibres ${ }^{* *}$. Following a description of the principles of construction and operation of the vertex detector and an outline of the experimental set-up used for tests in a particle beam, details are given of the analysis procedure devised to allow quantitative evaluation of target performance. This leads to determinations of the light output (hit density), the spatial resolution, and the severity of the noise. Estimates for more fundamental properties, such as relative intrinsic light yield and light attenuation within the fibres, are derived. There is a discussion of light transmission in narrow-diameter fibres and a consideration of the time

*) Levy Hill Laboratories, Ltd., 5 Sheffield House, Fieldings Road, Cheshunt, Waltham Cross, Herts. EN8 9TJ, UK.

**) Kyowa Gas Chemical Industry Co. Ltd., Shuwa Higashi-Yaesu Building, 1-Go, 9-Ban, 2-Chome, Hatchobori, Chuo-ku, Tokyo 104, Japan. 
response for the different scintillators. Final conclusions are drawn concerning the high-resolution tracking capabilities of the glass and plastic scintillating-fibre vertex detectors.

\section{EXPERIMENTAL SET-UP}

\subsection{The scintillating-fibre vertex detector}

The vertex detector is constructed using small-diameter $(\sim 30 \mu \mathrm{m})$ scintillating fibres, each consisting of a thin strand of scintillating material (core)-accounting for about $70 \%$ of the total volume - coated in a second material (cladding) of lower refractive index. Individual fibres are assembled in coherent fibre bundles ( $\leqslant 1 \mathrm{~mm}$ in diameter), from which a parallelepiped (or cylinder), having a cross-section of $\sim 15-25 \mathrm{~mm}^{2}$ and a length of a few centimetres, is formed. This device is an active target, acting simultaneously as target and vertex detector. It allows precise spatial measurements - to within a few tens of microns - in a region surrounding, and including, the primary vertex, viewed in a two-dimensional projection.

When charged particles cross the target, scintillation light is emitted isotropically at points along their paths. A small fraction of this light is trapped and propagates within the fibres, producing a two-dimensional image of the particle tracks at the target surface, as shown in fig. 1.

Readout is performed after recording the image using a charge-coupled device (CCD) ${ }^{*}$. Since a high light intensity is needed at the surface of the $\mathrm{CCD}$, the light pattern emerging at the target surface must be amplified. The optoelectronic chain that provides the image intensification (yielding about $10^{5}$ photons per photon), designed to operate in a region of high magnetic field $(\sim 1.8 \mathrm{~T})$, is briefly discussed in the next section. More detailed descriptions of the optoelectronic chain may be found in refs. [9] and [10].

\subsection{Set-up for tests in a particle beam}

In the standard set-up, the vertex detector is mounted in a light-tight aluminium box (box number 1), as shown in fig. 2, and placed in the beam line. The light emergent at one end of the target is piped from the beam line through a glass-fibre bent taper to the first stage of image intensification, performed by two proximity-focused image intensifiers (proxifiers) ${ }^{* *}$. The bent taper and the proxifiers are positioned, with the target, in box number 1 . The light image is then transported by a $2.5 \mathrm{~m}$ long image guide ${ }^{* * *}$, composed of $10 \mu \mathrm{m}$ diameter fibres and shielded by a black plastic covering, to a second light-tight aluminium box (box number 2), shown in fig. 3. Here, the strong light amplification necessary for the image to be recorded by the $\mathrm{CCD}$ is mostly provided by a gated microchannel-plate (MCP) image intensifier ${ }^{x}$. In order to preserve spatial resolution, the image is magnified by an electrostatically focused image intensifier ${ }^{\times} \times$), before it arrives at the entrance of the MCP. Demagnification is performed after the MCP, using a second electrostatically focused image intensifier ${ }^{\times \times \times)}$, so as to match the size of the CCD. The CCD consists of $550 \times 288$

*) Thomson-CSF, Division Tubes électroniques, 38 rue Vauthier, BP 305, 92102 BoulogneBillancourt Cedex, France.

**) Proxitronic GmbH, Robert Bosch Strasse 34, 6140 Bensheim, Fed. Rep. Germany.

***) Schott Fiber Optics, Inc., 122 Charlton Street, Southbridge, MA 01550, USA.

X) Electro-Optical Products Division, 3700 East Pontiac Street, PO Box 3700, Fort Wayne, Indiana 46801, USA.

$\times \times$ ) Varo Inc., Electron Devices Division, 2203 Walnut Street, P.O. Box 469014, Garland, Texas, USA.

$\times \times x)$ B.V. Delft Electronische Producten, Postbus 60, Dwazziewegen 2, 9300 AB Roden (Dr.), The Netherlands. 
pixels having dimensions of $16 \mu \mathrm{m} \times 23 \mu \mathrm{m}$. Overall magnification is such that a single fibre is viewed by $\sim 4$ pixels on the average. No important optical distortion is introduced by the optoelectronic chain.

Beam particles are able to enter and leave box number 1 through thin windows. For test purposes, coincidences in two narrow scintillation counters placed, orthogonal to one another, immediately upstream of the entrance window, and in a third scintillation counter positioned downstream from the target, formed a beam trigger which opened the gate of the MCP image intensifier. The gate was kept open for a specified time ( $4 \mu \mathrm{s}$ for the glass target tested and $1 \mu \mathrm{s}$ for the plastic targets) to allow the passage of light to the CCD. Following event readout at $4 \mathrm{MHz}$, a Fast Clear of the CCD was performed at a rate of $\geqslant 100 \mathrm{~Hz}$, using a technique (developed for the UA2 Collaboration [11]) which exploits the antiblooming facility.

The target could be rotated in the horizontal plane, so that the beam passed through the sides of the target, inclined at an angle $\theta$ with respect to the fibre axes. It was also possible to change the displacement of box number 1 , relative to the beam line, so that the distance between the beam entry position and the target end could be altered. The geometry of the setup is shown in fig. 4 .

\subsection{The active targets}

One target constructed from scintillating glass fibres ${ }^{*}$ and two made from scintillating plastic fibres have been tested at the CERN Proton Synchrotron, using the standard set-up and with a $6 \mathrm{GeV} / \mathrm{c}$ hadron beam.

The fibres from which the glass target was fabricated were square in cross-section, each consisting of a core of GS1 scintillator glass, containing 3\% (by weight) of the scintillator cerium (III) oxide $\left(\mathrm{Ce}_{2} \mathrm{O}_{3}\right)$, and a clear glass cladding of N51A. In addition, every fibre was coated with an EMA. Parallel sides of the core were separated by a distance of $25 \mu \mathrm{m}$, whilst the cladding had a thickness of $2 \mu \mathrm{m}$. The target had a $4 \mathrm{~mm} \times 4 \mathrm{~mm}$ square cross-section and a length of $4 \mathrm{~cm}$.

In constructing the plastic target, fibres of circular cross-section were used. However, in the course of the target fabrication process, which involved the drawing of bundles of fibres at high temperatures, the fibres were deformed in such a way that the cross-section became almost perfectly hexagonal. In the first of the plastic targets, the core material for the fibres was the scintillator SCSN-81T(0.50), where the number in parentheses indicates the percentage concentration (by weight) of the second wavelength shifter (WLS). This scintillator is similar to the standard scintillator SCSN-81, apart from the concentration of the second WLS, which is 25 times higher than usual. For the second plastic target, the core material for the fibres consisted of a polystyrene (PS) base doped with $1.5 \%$ (by weight) of a single WLS, 1-phenyl-3-mesityl-2-pyrazoline (PMP), having a large Stokes' shift [7]. In both cases, the fibre cladding was polymethyl methacrylate (PMMA) and the dimensions were the same; parallel sides of the hexagonal cores were separated by a distance of $30 \mu \mathrm{m}$, whilst the cladding thickness was $3 \mu \mathrm{m}$. Although no EMA could be placed about the individual fibres, the targets themselves were coated with an absorbent black paint, so as to avoid the uniformly distributed noise that would have resulted from total internal reflection of the untrapped light at an air-plastic boundary. Each of the plastic targets had a $5 \mathrm{~mm}$ diameter circular cross-section and a length of $5 \mathrm{~cm}$.

The target dimensions are those that are believed appropriate for the study of heavy-particle decays, allowing sufficient material for the observation of the decays while avoiding an excessive number of secondary interactions.

*) Collimated Holes, Inc. (R.W. Mead, President), 460 Division Street, Campbell, CA 95008, USA. 
The physical properties of the glass and plastic scintillating fibres used in target construction, including some of those measured by the WA84 Collaboration and detailed in the following sections, are summarized in table 1 .

\section{RESULTS OF TESTS IN A PARTICLE BEAM}

\subsection{Data recorded and analysis procedure}

Ideally, it would have been desirable to have recorded particle tracks with the target axis rotated by $90^{\circ}$ with respect to the beam line $\left(\theta=90^{\circ}\right.$ in fig. 4), whereby the distance travelled by the scintillation light before detection would have been the same in all fibres. However, the amount of light emitted per unit path length traversed by a minimum-ionizing particle is generally too low to ensure unambiguously defined tracks under such conditions, given the small cross-sections of the targets. In order to increase the distance travelled by a beam particle within each fibre, so that an acceptable amount of light was generated, data were taken with $\theta=12^{\circ}$ for the glass target and with $\theta=20^{\circ}$ for the plastic targets. For the data samples for which results are given in subsections 3.2, 3.3 , and 3.4, the beam line passed through the target centre. In each case, around 300 triggers were recorded. Figures 5, 6, and 7 show the light patterns produced by particles crossing the different targets.

Each photoelectron detected at the phosphor of the first image intensifier appears on the CCD as a cluster of pixel-hits (pixels having non-zero pulse heights) and is referred to as a hit or spot. In the analysis, a hit could be identified as a local maximum in the pulse height in a region of at least 3 $\times 3$ pixels. Isolated pixel-hits, however, were attributed to low-level electronic noise and were disregarded. The probability of two hits being superimposed in such a way that they became impossible to resolve, under the experimental conditions chosen, was negligible. An initial filtering of events was performed so as to reject empty frames, frames dominated by unusually high levels of noise, and frames containing more than one particle track.

Two methods were used for track reconstruction. Both of these started from a consideration of the pixel-hits in a region having a width of $800 \mu \mathrm{m}$ (as measured at the target surface) in which, based on the distribution of pulse heights over the frame, the particle track was expected to be found. In the first method, a linear least-squares fit was made to the pixel-hits, where each pixel coordinate was weighted by the pixel pulse height. The second method differed from the first in that the pixel-hits were allocated to spots by clustering. An unweighted linear least-squares fit was then made to the coordinates of the spot barycentres. In both cases, the quality of the fit was improved iteratively by starting from the already fitted line and refitting to points within an increasingly narrow region around the line. Well-defined tracks were selected by requiring that the length and the number of points used in the final iteration of fitting be above some minimum values. Results given by the two methods are consistent with one another and are not distinguished here.

The aim of the analysis carried out on the chosen tracks was to provide an objective assessment of target performance. This was achieved by determining the spatial resolution, the light output (measured in terms of a hit density), and the contributions from noise to the pulse height on an image frame.

\subsection{Spatial resolution}

The spatial resolution of the detector can be described in terms of three parameters. These are the spot size, the root-mean-square track residual, and the two-track resolution. The third of these quantities is determined by the other two. All measurements made are with reference to the two-dimensional image projection at the target surface. 


\subsubsection{Spot size}

An isolated spot is due to a single photoelectron detected at the phosphor of the first image intensifier, so that the spot size is essentially unaffected by the target used and depends only on the spatial resolution of the components of the optoelectronic chain that come after the bent taper. The spot profile is found by superimposing the barycentres of many spots and projecting the pixel pulse heights onto two orthogonal axes (labelled $\mathrm{x}$ and $\mathrm{y}$ ). Spot profiles obtained for the glass target are shown in fig. 8. Root-mean-square values $\sigma_{\mathrm{Sx}}$ and $\sigma_{\mathrm{Sy}}$ for the spot profiles, which measure the spot size, are given in columns two and three of table 2 . The spot profiles for the glass target are, in fact, marginally wider than those obtained for the other targets (not shown). This small effect is believed to be due to a slight saturation in the CCD preamplifier at the time that the data for the glass target were recorded, later corrected.

\subsubsection{Root-mean-square track residual}

The track residual measures the displacement of a hit from the fitted track. Distributions of track residuals for the tracks seen in the three targets are shown in fig. 9. After background subtraction, each distribution is approximately Gaussian in form, and the root-mean-square track residual $\sigma_{\mathrm{tr}}$ may be calculated. Values for $\sigma_{\mathrm{tr}}$ are given in column four of table 2 . The root-meansquare track residual of $19 \mu \mathrm{m}$ measured for the glass target is consistent with that of ref. [12].

A hit may be displaced from a track for a number of reasons, predominantly relating to the target used. More particularly, there are contributions to $\sigma_{\mathrm{tr}}$ which are due to the fibre size, to any target incoherency (resulting from non-perfect parallel alignment of fibres), to the presence of delta rays, to Coulomb scattering of the beam particles, to distortion in the bent taper and image intensifiers, and to $\mathrm{CCD}$ digitization uncertainty.

\subsubsection{Two-track resolution}

The combined effect on the spatial resolution of the sizes of the spots and of their displacements from a track is seen by histogramming, for all tracks, the perpendicular distance of each pixel from the fitted line, weighted by the pixel pulse height. This gives the distribution of pulse heights transverse to the track direction. Such distributions are shown in fig. 10 for the three targets. The two-track resolution is measured by the root-mean-square values, $\sigma_{\mathrm{tt}}$, calculated after background subtraction. Results are given in the fifth column of table 2.

\subsection{Hit density}

The number of detected hits is proportional to the amount of light arriving at the target end, where the constant of proportionality is determined by the fraction of light transmitted between the target and the first proxifier and by the single-photon detection efficiency of the first proxifier.

For the purposes of the analysis, a hit is considered to be associated with the fitted track if it is displaced by a distance no greater than $3 \sigma_{\mathrm{tt}} \approx 60 \mu \mathrm{m}$. The hit density is then defined as the ratio of such track-associated hits to the track length in the target. Experimentally measured values of hit densities are given in table 3 . Statistical errors are negligible, but it is estimated that systematic errors could be as high as \pm 0.2 hits per $\mathrm{mm}$.

The hit density found experimentally is dependent on the scintillation yield of the material used for the fibre core and on the light attenuation within the fibres. Since the amount by which the light is attenuated is determined by the distance it travels, the measured hit density along a straight-line particle track will depend on the positions at which the particle enters and leaves the target. In view of this fact, the hit densities quoted in table 3, for particles passing through the target centres, should be considered only as representative values. These are useful for making comparisons. 
The hit densities obtained with the plastic fibres are more than twice the hit density obtained with the glass fibres. As will be shown later (subsections 4.1 and 4.3), this is due to the much longer light attenuation lengths in the plastic fibres rather than to any great dissimilarity in the intrinsic light yields per unit path length traversed by a minimum-ionizing particle. In the case of the target constructed from PMP fibres, the attenuation length is sufficiently long for a mirrored surface placed at the non-readout end to produce a beneficial increase in the hit density. Experimentally, with an aluminized Mylar screen as mirror, hit densities of $2.3 \mathrm{~mm}^{-1}$ have been observed. Optical coupling losses between the Mylar screen and the target can be considerable, so that the hit density should be further increased by directly aluminizing the target end. Only a slight improvement in the hit density is measured when the mirror is used with the target of SCSN-81T(0.50) fibres, and for the glass target a mirror has no effect.

\subsection{Noise}

The contributions from noise to the total light detected are evaluated by considering the distributions of pulse height transverse to the track direction (fig. 10), again as seen in the two-dimensional projection at the target surface. In analogy with the definition for track-associated hits, the track-associated pulse height is defined as the sum of the pulse heights of the pixels within $3 \sigma_{\mathrm{tt}}$ of the fitted track. The remaining pulse heights (including those due to pixel-hits further than $200 \mu \mathrm{m}$ from the track, not histogrammed) are attributed to some form of noise. Integrated over the entire image frame, the contribution of the noise to the total light detected is $27 \%$ for the glass target and $40 \%$ for each of the plastic targets.

The majority of hits detected far from the track are indistinguishable from the track-associated hits, indicating that the noise originates in or before the first image intensifier. A series of tests has been performed to investigate the sources of noise, with the aim of reducing their contributions. The findings of these tests are reported in a separate paper [13] and are only mentioned here.

Part of the noise (experimentally measured to be $\sim 13 \%$ of the track-associated pulse height on a frame) is generated in the optoelectronic chain and is mostly due to the backscattering of electrons at the phosphor of the first proxifier. In the presence of a strong magnetic field, such as is to be used for the WA84 experiment (but absent for the tests for which results are presented here), this noise should be concentrated about the particle tracks, becoming essentially unimportant for the pattern recognition.

There is a contribution from delta rays with ranges greater than $3 \sigma_{\mathrm{tt}}$ (calculated to be $-10 \%$ of the total pulse height on a frame for both the glass and plastic targets). This contribution cannot be removed and places a physical limitation on the performance levels that can be attained using a scintillating-fibre vertex detector.

The remaining noise is a consequence of light being detected in a fibre other than that in which the primary emission takes place; this is termed cross-talk. Possible cross-talk mechanisms are still being studied, but some of them have been identified and understood. These are further discussed in Section 5.

In table 4, estimates of the various contributions to the light seen on an image frame are given for the three targets. The differences in the amounts of cross-talk for the glass and plastic targets are essentially due to the absence of an EMA coating on single plastic fibres. From the non-zero level of cross-talk in the glass target, it is evident that the very thin layer of absorbent material on each individual fibre is not completely opaque. Results from the tests of noise reduction methods show that an improvement in the signal-to-noise ratio is obtained for plastic targets by assembling them from black-coated fibre bundles having cross-sections of $1 \mathrm{~mm} \times 1 \mathrm{~mm}$ or less. A decrease from $\sim 40 \%$ to $\sim 25 \%$ in the contribution of the noise to the total light has been measured, corresponding to a strong reduction of the cross-talk. 


\section{OTHER RESULTS}

\subsection{Light attenuation in fibres}

The attenuation by a scintillator of its own emitted light is non-exponential, but may be described by $\sim \exp [-z / \lambda(z)]$, where the light losses at a distance $z$ from the point of emission are characterized by an attenuation length $\lambda(z)$. As demonstrated for example in ref. [4], for GS1 glass, the measured attenuation length is markedly lower at shorter distances. However, over the small intervals of interest in the fibres tested it is assumed that $\lambda(z)=\lambda_{0}$ is approximately constant. The values for the attenuation lengths $\lambda_{0}$ in the fibres, determined from the track data, are given in table 5. Two equivalent methods were used in calculating these attenuation lengths.

In the first method, tracks recorded under fixed conditions, with the target axis at an angle $\theta$ with respect to the beam line, were considered. For each track, the light output was plotted as a function of the distance $\mathrm{z}$ between the point of light emission and the surface of detection, determined from the position along the track. The resulting curve could then be fitted with an exponential $\sim \exp \left(-z / \lambda_{0}\right)$. This method is found to be reliable in the cases where there is significant light attenuation in the interval $\ell_{0} / \tan \theta$, where $\ell_{0}$ is the track length measured at the target surface. An attenuation curve thus obtained is shown in fig. 11 for the glass target, in which the light transmission is extremely poor.

In the second method, tracks recorded with the beam entering at different distances $\mathrm{z}_{0}$ from the target end (but with the angle between the beam line and the target axis fixed) were considered. The total mean light output per track was then plotted as a function of the beam entry position, producing a curve fitted by the exponential $\sim \exp \left(-z_{0} / \lambda_{0}\right)$. An attenuation curve obtained using this method is shown in fig. 12 for the target constructed from SCSN-81T(0.50) fibres.

The light attenuation length in the PMP fibres was too long to be accurately measured in the standard-size target. For this reason, tracks were recorded also in a second target of PMP fibres, having a length of $200 \mathrm{~mm}$ but otherwise the same as the first, and using a slightly non-standard set-up (necessitated by the mechanical requirements for the mounting of the target). The attenuation curve for this target is shown in fig. 13.

No reflective surfaces were positioned at the target ends for the determination of light attenuation lengths.

\subsection{Decay time of light emission}

Measurements of the decay times $\tau_{\mathrm{i}}$ of light emission have been reported by the WA84 Collaboration for GS1 glass fibres [6], and by another group for a small number of scintillating plastic fibres [14]. In all cases, the rate of light emission $\mathrm{dI}(\mathrm{t}) / \mathrm{dt}$ has been found to be well described by expressions having the form:

$$
\frac{\mathrm{dI}}{\mathrm{dt}}(\mathrm{t})=\sum_{\mathrm{i}} \frac{\mathrm{A}_{\mathbf{i}}}{\tau_{\mathrm{i}}} \exp \left(-\mathrm{t} / \tau_{\mathrm{i}}\right)
$$

Values obtained for the decay times of different components, and their relative intensities $A_{i}$, are summarized in table 6. The existence of the long-lived components of the GS1 light emission have evident consequences for a gated system (such as the one considered here), imposing severe restrictions on the event rates that may be sustained.

In fig. 14, the integrated light intensity at the gate of the MCP image intensifier is plotted as a function of the time interval following the commencement of light emission. The time response of the part of the optoelectronic chain that precedes the MCP image intensifier is taken into account by considering i) the responses of the $\mathrm{X} 3$ phosphors of the two proxifiers and of the $\mathrm{P} 47$ phosphor [15] of the first of the electrostatically focused image intensifiers (for which decay times for the light emission are also given in table 6$)$ and ii) the delay ( $\sim 15 \mathrm{~ns})$ introduced by the image guide. 
For the plastic fibres, some $10 \%$ of the light arriving at the MCP image intensifier is lost during the interval $(\sim 100 \mathrm{~ns})$ before the gate can be opened. All remaining light can be recorded with a gate length of about $1 \mu \mathrm{s}$. This means that, in conjunction with the optoelectonic chain currently employed, these fibres are ideal for use in particle detection in experiments for which the event rate is $\leqslant 1 \mathrm{MHz}$. The limitation is a consequence of the temporal dispersion of the light signal by the optoelectronic chain. It should be noted that the light which is lost because of delay in opening the gate (or because of a short gate length) is essentially only a fraction of the light which arrives at the MCP over several hundreds of nanoseconds, and which is due to each of the single photoelectrons released, in a time interval of a few nanoseconds, at the photocathode of the first proxifier. The loss in light does not correspond to a loss of hits but to a loss of pulse height.

For the GS1 glass fibres, less than $2 \%$ of the light arriving at the MCP is lost before the gate is opened. However, the integration time necessary for recording the remaining light is greater by several orders of magnitude than that needed when using the plastic fibres. In this case, the use of a gate length of the order of $1 \mu \mathrm{s}$ has the effect of reducing the number of hits detected, because the light continues to be emitted by the scintillator long after the initial excitation. The superposition of images due to interactions in the target occurring within less than $\sim 100 \mu \mathrm{s}$ of one another is inevitable, so that the GS1 fibres are unsuitable for use in experiments in which the event rate is greater than about $10 \mathrm{kHz}$.

\subsection{Light yield}

In previous papers $[4,16]$ the light yield for GS1 glass has been quoted as $1.5 \pm 0.5$ photons per $\mathrm{keV}$ for minimum-ionizing particles (without reference to the light integration time). Measurements by the WA84 Collaboration [6] indicate that this value is consistent with the light yield of only the fast component of the light decay, and that the total light yield, integrated over an extended time interval, is $\sim 5$ photons per $\mathrm{keV}$. This is to be compared with $\sim 9$ photons per $\mathrm{keV}$ for the plastic scintillators used. Taking into account the differences in energy deposition for minimum-ionizing particles in glass and plastic, the light yield per unit path length traversed by the particle is then higher (by a factor of about 1.3) for the GS1 glass than for the plastic scintillators. The same conclusion is reached from a consideration of the experimentally measured hit densities.

A particle that enters an active target at a distance $z_{0}$ from the surface of detection, travelling along a path inclined at an angle $\theta$ to the fibre axes, produces a track of length $\ell_{0}$ at the target surface, defined by I hits (fig. 4). Assuming an exponential light attenuation within the fibres, characterized by a constant attenuation length $\lambda_{0}$, it may be shown that:

$$
\mathrm{I}=\frac{\mathrm{dI}}{\mathrm{d} \ell} \frac{\lambda_{0}}{\cos \theta} \mathrm{e}^{-\mathrm{z}_{0} / \lambda_{0}}\left[1-\mathrm{e}^{\left.-\ell_{0} / \lambda_{0} \tan \theta\right)}\right]
$$

where $\mathrm{dI}_{0} / \mathrm{d} \ell$ represents the hit density which would be observed in the absence of attenuation, and which is proportional to the intrinsic light yield. Values of $\mathrm{dI}_{0} / \mathrm{d} \ell$ for the glass and plastic fibres tested, determined from the results obtained under experimental conditions, are given in table 7 . The sensitivity of the photocathode of the first image intensifier was approximately the same for the emission spectra of the three fibre types. However, the value given for the GS1 glass has been corrected, since only $70 \%$ of the scintillation light would have been collected in the $4 \mu \mathrm{s}$ integration time used. Notwithstanding the many possible sources of error, the photoelectron yield per unit length is indeed seen to be slightly larger for the GS1 than for the plastics (by a factor of 1.2, where a small correction is made to allow for the fact that the fraction of light initially trapped is a little different for the glass and plastic fibres, as detailed in the next section). 
It is interesting to note that even in the absence of light attenuation in the targets, only $\sim 0.1 \%$ of the scintillation light would be detected. This figure is arrived at by considering the packing fraction of the target $(\sim 0.70)$, the fraction of light trapped and propagated in the fibres $(\sim 0.03$, limited by the differences in the refractive indices of core and cladding materials and by the fibre geometry), the transmission efficiency of the bent taper $(\sim 0.40)$, the quantum efficiency of the photocathode of the first proxifier $(\sim 0.16$, after taking into account the transparency of the input fibre window), and the likelihood of a photoelectron being detected at the first phosphor $(\sim 0.60$, suggested by ref. [17]).

Also of relevance - and particularly in the study of high-energy interactions - are the light yields of the scintillators for heavily ionizing particles. After normalizing to the same energy deposition, the WA84 Collaboration finds [6] that the ratio of the light yield for a $3.65 \mathrm{MeV}$ alpha particle to that of a minimum-ionizing particle is $0.24 \pm 0.03$ for GS1 and $0.10 \pm 0.02$ for NE102A plastic scintillator. When high-energy interactions are taking place, heavily ionizing particles produced during nuclear evaporation may cause obscuration about the interaction vertex. For a plastic target, as compared to a glass one, the stronger saturation in the light emission, together with the lower multiplicity of heavily ionizing particles, should reduce any such obscuration, leading to superior resolution in the region of the vertex.

\section{LIGHT TRANSMISSION IN NARROW-DIAMETER FIBRES}

The fraction of the isotropically emitted scintillation light that is trapped in the fibre in which it is produced - referred to as the trapping fraction - is determined by the ratio between the indices of refraction for core and cladding and by the geometry of the fibre cross-section ${ }^{*}$. Based on considerations of geometry alone, the trapping fraction is higher in fibres with square cross-section than in those with hexagonal cross-section. Monte Carlo simulations of light propagation in square and hexagonal fibres [18], in which perfectly smooth core-cladding boundaries are assumed, indicate a trapping fraction of 0.032 in each direction in the glass microfibres tested, and of 0.034 in each direction in the plastic microfibres. The greater difference in the indices of refraction of the core and cladding materials compensates for the less efficient trapping of the hexagonal cross-section. Trapped light is transmitted along the fibres through a series of (near) total internal reflections.

In the GS1 bulk glass, about $45 \%$ of the scintillation light is lost in the first $2 \mathrm{~cm}$, as illustrated in ref. [16], because of the overlap of the emission and absorption spectra. The Stokes' shift is wide enough to allow the remaining light to be transmitted without being significantly reabsorbed. By contrast, for bulk plastic scintillators there is considerable overlap between the spectra for emission and absorption. The standard solution to this problem is to dope the scintillator with an additional WLS, which absorbs the light emitted by the primary solute and re-emits it at longer wavelengths. An advantageous secondary effect is that the shifted spectra thus obtained are better matched to the

*) In an ideal fibre, having core and cladding with indices of refraction $n_{c o}$ and $n_{c l}$ respectively, in which scintillation light is produced isotropically and uniformly in the core, the trapping fraction in each direction along the fibre is exactly

$0.5\left(1-\mathrm{n}_{\mathrm{cl}}^{2} / \mathrm{n}_{\mathrm{co}}^{2}\right)$

for circular cross-section and

$\frac{2}{\pi} \int_{0}^{\pi / 4}\left\{1-\left[1-\frac{1-\mathrm{n}_{\mathrm{c}}^{2} / \mathrm{n}_{\mathrm{co}}^{2}}{\cos ^{2} \phi}\right]^{1 / 2}\right\} \mathrm{d} \phi$

for square cross-section. 
spectral sensitivities of existing photocathodes. The difficulties in optimizing the concentrations of the dopants, so as to obtain both emission that is local to the point of energy deposition (essential for microfibres) and efficient light transmission, are discussed, for example, in ref. [19]. In the case of short-length fibres at least, these problems of chemical composition are essentially solved. This has been achieved in two ways. In SCSN-81T(0.50), the concentration of the second WLS is 25 times higher than usual so that absorption of the primary light occurs within $\sim 10 \mu \mathrm{m}$ of emission, as compared with $\sim 300 \mu \mathrm{m}$ in a standard plastic scintillator. For fibres having a PS [or polyvinyl toluene (PVT)] base doped only with PMP, the large Stokes' shift allows the light to be transmitted, with little reabsorption.

Light attenuation in fibres depends on the intrinsic absorption in the bulk material and on the reflection losses at the core-cladding interface (where at present the reflection coefficient is typically no better than 0.9995). For the small-diameter fibres considered, the number of reflections increases strongly with the angle between the direction of light propagation and the fibre axes. Light travelling at larger angles is, then, more rapidly attenuated. For GS1 glass, it has been demonstrated [4] that at short distances $(\leqslant 2 \mathrm{~cm}$ ) from the point of light emission, where losses due to the overlap of the absorption and emission spectra are dominant, there is little difference between the attenuation in the fibres and in the bulk material. At intermediate distances (between $2 \mathrm{~cm}$ and $40 \mathrm{~cm}$ ) there are significant losses for light being reflected internally at the core-cladding boundary in narrow-diameter fibres, so that the attenuation in the fibres is greater than in the bulk material. Eventually (for distances $\geqslant 40 \mathrm{~cm}$ ), all but the most forward-going light in the fibres is dissipated, having undergone multiple reflections, and the attenuation in the fibres is again the same as in the bulk material. The severity of the reflection losses is determined by the quality of the core-cladding interface, which is dependent on the manufacturing process.

Considerations of the quality of the core-cladding interface, of the fibre geometry, and of the properties and concentrations of the dopants are also relevant to an understanding of the cross-talk mechanisms.

Imperfections at the core-cladding boundary can cause the light that would otherwise be transmitted to neighbouring fibres to be scattered in such a way that it is trapped and detected as noise. For a good quality interface, this kind of cross-talk becomes negligible.

Depending on the geometry of the fibre cross-sections, light initially emitted at large angles with respect to the fibre axes may remain trapped in a system of fibres, or in a single fibre, after being refracted and reflected many times. Monte Carlo studies have shown that the fraction of the light that is trapped in this way decreases with the distance from the point of emission. It is less, by almost one order of magnitude, for fibres of square cross-section than for those of hexagonal cross-section, becoming negligible for the lengths of glass fibres tested, although not for the plastic fibres.

Another form of cross-talk is present when there is an overlap of the absorption and emission spectra of the scintillator. This allows the absorption by the scintillator of the untrapped scintillation light (accounting for more than $90 \%$ of the total), after which isotropic re-emission may occur. A certain percentage of the re-emitted light is then trapped, generating cross-talk. Since the light can travel a long distance before it is absorbed, the cross-talk produced by this mechanism can have a considerable range.

In principle, the use of a second WLS in plastic fibres can give rise to further cross-talk when light is emitted by the primary solute in one fibre but undergoes the shift in wavelength in a different fibre. This kind of cross-talk is found to be negligible in the SCSN-81T(0.50) fibres, with the high concentration of the second WLS, and the total amount of cross-talk is seen to be the same as for the PMP-doped fibres, in which there is only the single WLS.

The effect of the concentration of the second WLS in a plastic scintillator is illustrated in fig. 15 , where the light output of the standard SCSN-81T fibres [containing $0.02 \%$ (by weight) of the second 
WLS] may be compared with the light outputs for the SCSN-81T(0.50) fibres and for the PMP-doped PS fibres. Black-painted bundles of the different fibre types, having $1 \mathrm{~mm}$ diameter circular cross-sections, were assembled to form a composite target, and the light due to $6 \mathrm{GeV} / \mathrm{c}$ hadrons passing through the three bundles was recorded. Individual fibres were hexagonal in cross-section, with parallel sides separated by a distance of $30 \mu \mathrm{m}$, as before. The primary light yield for the standard SCSN-81T fibres is the same as for the SCSN-81T(0.50) fibres. However, because of the low concentration of the second WLS, the mean distance travelled by the primary light before it can be wavelength-shifted is greater, and the fraction of the primary light that is absorbed (in the scintillator or at the black-painted outer surface) is higher. The lower detected light output for the standard SCSN-81T fibres then reflects the fact that the final shift in wavelength is non-local to the point of excitation.

\section{CONCLUSIONS}

It has been shown that active targets constructed using narrow-diameter $(\sim 30 \mu \mathrm{m})$ scintillating fibres can be used as high-resolution tracking detectors, giving a two-track resolution $\sigma_{\mathrm{Tr}} \approx 20 \mu \mathrm{m}$ for the tracks analysed.

Cerium-doped GS1 glass fibres can be assembled to provide precise tracking detectors, of dense material, which are highly radiation-resistant. The long light-emission time of the scintillator $(\geqslant 1 \mathrm{~ms}$ for all light) restricts the use of such detectors to situations in which only low event rates $(\leqslant 10 \mathrm{kHz})$ are required, as in neutrino experiments for example. Although the light yield per unit path length traversed by a minimum-ionizing particle in the GS1 fibres is relatively high, a substantial fraction is lost because of the poor light-transmission properties, giving an observed hit density of only $0.6 \mathrm{~mm}^{-1}$. An increase in the light attenuation length, entailing improvements in the fibre manufacturing process, would be essential for these fibres to have any practical application.

Detectors constructed from either SCSN-81T(0.50) or PMP-doped plastic fibres have the advantages of a fast time response and a reasonable light-transmission efficiency. With the optoelectronic chain built for the WA84 experiment, all track information can be recorded with a light integration time of less than $1 \mu \mathrm{s}$, so that the targets are ideally suited to experiments with event rates of $\sim 1 \mathrm{MHz}$. For minimum-ionizing particles, hit densities as high as $2.3 \mathrm{~mm}^{-1}$ have been observed (in a target of PMP-doped plastic fibres having a reflective surface at the non-readout end). The disadvantage of plastic targets without any EMA is that the amount of noise generated is large (about $40 \%$ of all light detected, spread over the entire image frame). This noise will be much reduced when it becomes possible to coat the individual plastic fibres with an EMA before target fabrication, in a procedure analogous to that already employed in the construction of glass-fibre targets. Measurements by the WA84 Collaboration [13] have suggested more immediate ways of increasing the signal-to-noise ratio. These should reduce the contribution of the noise to no more than $\sim 15-20 \%$ of the total light detected, an ultimate limit of $\sim 10 \%$ of the total being imposed by delta-ray production. The noise reduction methods are being implemented and, using the plastic active targets, the efficiency of the detector system for the study of high-energy interactions will be tested.

\section{Acknowledgements}

The support of Drs. H. Wenninger and J.C. Gouache (EF Division, CERN) during the development of this project is gratefully acknowledged. We are indebted to the engineers and technicians of the EF Instrumentation Group who have contributed to this work, and in particular to J. Dupont and J. Dupraz for their continued involvement. 


\section{REFERENCES}

[1] G.T. Reynolds and P.E. Condon, Rev. Sci. Instrum. 28 (1957) 1098.

[2] W. Beusch et al., proposal CERN/SPSC 87-2, SPSC/P 226 (1987).

[3] W. Beusch et al., technical report CERN/SPSC 77-70, SPSC/T-17 (1977).

[4] M. Atkinson et al., Nucl. Instrum. Methods A254 (1987) 500.

[5] R. Ruchti et al., IEEE Trans. Nucl. Sci. NS-33 (1986) 151.

[6] C. Angelini et al., Nucl. Instrum. Methods A281 (1989) 50.

[7] P. Destruel et al., Nucl. Instrum. Methods A276 (1989) 69.

[8] H. Blumenfeld et al., Nucl. Instrum. Methods A278 (1989) 619.

[9] C. Angelini et al., Nucl. Instrum. Methods A277 (1989) 132.

[10] C. Roda, Tesi di laurea, Università di Pisa, 1989 [report CERN/EF/INSTR 89-1 (1989)].

[11] S. Reynaud, report CERN/EF/INSTR 86-1 (1986).

[12] M.N. Atkinson et al., Nucl. Instrum. Methods A263 (1988) 333.

[13] C. Angelini et al., Sources of noise in high-resolution tracking with scintillating fibres, presented by G. Penso at the 4th Pisa Meeting on Advanced Detectors, La Biodola, Isola d'Elba, 1989, Proceedings to be published in Nucl. Instrum. Methods.

[14] M. Kuhlen, CalTech report CALT-68-1547 (1989), presented at Int. Industrial Symp. on the Super Collider, New Orleans, LA, 1989.

[15] Optical characteristics of cathode-ray tube screens, formulated by EIA Tube Engineering Advisory Council (TEPAC), TEPAC publication 116 (1980).

[16] J. Kirkby, preprint CERN-EP/87-60 (1987), Proc. Workshop for the INFN ELOISATRON Project: Vertex detectors, Erice, 1986 (Plenum Press, New York, 1988), p. 225.

[17] R.H. Cromwell, Toward solving the lost photon problem in image intensifiers, internal report Steward Observatory, University of Arizona.

[18] G. Wilquet, private communication.

[19] A. Grimes et al., Northeastern Univ. report NUB-2943 (1988). 
Table 1

Physical properties of glass and plastic scintillating fibres

\begin{tabular}{|llll|}
\hline $\begin{array}{l}\text { Fibre core: } \\
\text { Material }\end{array}$ & GS1 & SCSN-81T(0.50) & $\begin{array}{l}\text { PMP-doped } \\
\text { polystyrene }\end{array}$ \\
Density $\left(\mathrm{g} / \mathrm{cm}^{3}\right.$ ) & & & 1.06 \\
Shape of cross-section & 2.64 & 1.06 & Hexagonal \\
Width (distance between parallel sides) $(\mu \mathrm{m})$ & Square & Hexagonal & 30 \\
Mean refractive index (emission spectrum) & 25 & 30 & 1.59 \\
Radiation length (cm) & 1.59 & 1.59 & 41.3 \\
Interaction length (cm) & 9.77 & 41.3 & 77.4 \\
dE/d $\ell$ for minimum-ionizing particle (MeV/cm) & 4.6 & 7.4 & 2.0 \\
Light decay-time (fast component) (ns) & 71 & 2.4 & 3.0 \\
Percentage of light emitted by fast decay (\%) & 31 & 79 & $\sim 80$ \\
Photon yield (photons per keV) & $5.0 \pm 0.5$ & $9.0 \pm 2.0$ & $9.0 \pm 2.0$ \\
Wavelength of peak emission (nm) & 395 & 446 & 425 \\
Attenuation length measured in fibres (cm) & 2.0 & 9.4 & 30.8 \\
& & & \\
Fibre cladding: & & & PMMA \\
Material & N51A & PMMA & 3 \\
Thickness per micron & 2 & 3 & 1.49 \\
Mean refractive index (emission spectrum) & 1.51 & 1.49 & No \\
EMA present & & & \\
\hline
\end{tabular}

Table 2

Measurements of spatial resolution

\begin{tabular}{|l|c|c|c|c|}
\hline Fibre core & $\begin{array}{c}\text { Spot size } \\
(\mathrm{x} \text { projection) } \\
\sigma_{\mathrm{Sx}} \\
(\mu \mathrm{m})\end{array}$ & $\begin{array}{c}\text { Spot size } \\
(\mathrm{y} \text { projection) } \\
\sigma_{\mathrm{Sy}} \\
(\mu \mathrm{m})\end{array}$ & $\begin{array}{c}\text { Track residual } \\
\sigma_{\mathrm{tr}} \\
(\mu \mathrm{m})\end{array}$ & $\begin{array}{c}\text { Two-track } \\
\text { resolution } \\
\sigma_{\mathrm{tt}} \\
(\mu \mathrm{m})\end{array}$ \\
\hline GS1 & 13 & 14 & 18 & 23 \\
SCSN-81T(0.50) & 11 & 12 & 19 & 22 \\
PMP-doped polystyrene & 11 & 10 & 18 & 20 \\
\hline
\end{tabular}




\section{Table 3}

Number of experimentally measured hits per unit path length traversed by a minimum-ionizing particle (hit density). Particles passed through the centre of the target. The fibre axes were rotated by $12^{\circ}$ with respect to the beam line for the glass target and by $20^{\circ}$ for the plastic targets.

\begin{tabular}{|l|c|c|}
\hline \multicolumn{1}{|c|}{ Fibre core } & $\begin{array}{c}\text { Hit density } \\
\left(\mathrm{mm}^{-1}\right)\end{array}$ & $\begin{array}{c}\text { Mirrored surface } \\
\text { at non-readout end of target }\end{array}$ \\
\hline GS1 & 0.6 & No \\
SCSN-81T(0.50) & 1.4 & No \\
PMP-doped polystyrene & 1.6 & No \\
PMP-doped polystyrene & 2.3 & Yes \\
\hline
\end{tabular}

\section{Table 4}

Relative contributions to the pulse height on an image frame. Cross-talk is lower in the glass target because each fibre was coated with an EMA.

For the plastic targets, only the outer surfaces were painted black.

\begin{tabular}{|l|c|c|c|c|}
\hline \multicolumn{1}{|c|}{ Fibre core } & $\begin{array}{c}\text { Track-associated } \\
\text { pulse height }\end{array}$ & $\begin{array}{c}\text { Pulse height } \\
\text { due to } \\
\text { cross-talk }\end{array}$ & $\begin{array}{c}\text { Pulse height } \\
\text { due to } \\
\text { delta-rays }\end{array}$ & $\begin{array}{c}\text { Pulse height } \\
\text { due to } \\
\text { electronic noise }\end{array}$ \\
\hline GS1 & 0.73 & 0.08 & 0.10 & 0.09 \\
SCSN-81T(0.50) & 0.60 & 0.22 & 0.10 & 0.08 \\
PMP-doped polystyrene & 0.60 & 0.22 & 0.10 & 0.08 \\
\hline
\end{tabular}

Table 5

Light-attenuation lengths measured in fibres

\begin{tabular}{|l|c|}
\hline \multicolumn{1}{|c|}{ Fibre core } & $\begin{array}{c}\text { Attenuation length } \\
(\mathrm{cm})\end{array}$ \\
\hline GS1 & 2.0 \\
SCSN-81T(0.50) & 9.4 \\
PMP-doped polystyrene & 30.8 \\
\hline
\end{tabular}


Table 6

Different contributions to the light emission of scintillating fibres and image intensifier phospors

\begin{tabular}{|c|c|c|c|}
\hline Light emitter & $\begin{array}{l}\text { No. of components } \\
\text { considered }\end{array}$ & $\begin{array}{l}\text { Component } \\
\text { decay-time } \\
\text { (ns) }\end{array}$ & $\begin{array}{l}\text { Relative } \\
\text { intensity }\end{array}$ \\
\hline \multirow[t]{5}{*}{$\mathrm{GS}^{\mathrm{a})}$} & 5 & 71 & 0.305 \\
\hline & & 264 & 0.264 \\
\hline & & 2490 & 0.155 \\
\hline & & 18600 & 0.118 \\
\hline & & 349000 & 0.158 \\
\hline \multirow[t]{2}{*}{ SCSN-81 ${ }^{\text {b) }}$} & 2 & 2.4 & 0.787 \\
\hline & & 14.2 & 0.213 \\
\hline \multirow[t]{3}{*}{ X3 phosphor ${ }^{c)}$} & 3 & 44 & 0.502 \\
\hline & & 78 & 0.320 \\
\hline & & 190 & 0.178 \\
\hline P47 phosphor ${ }^{d)}$ & 1 & 38 & 1.000 \\
\hline
\end{tabular}

a) Values from ref. [6].

b) Values from ref. [14], with rise-times neglected. The response of the plastic fibres tested is similar.

c) Values from fit to data supplied by Proxitronic $\mathrm{GmbH}$.

d) Values from fit to data contained in ref. [15].

Table 7

Number of hits that would be detected per unit path length traversed by a minimum-ionizing particle, integrating over all light, in the absence of attenuation in the target

\begin{tabular}{|l|c|}
\hline \multicolumn{1}{|c|}{ Fibre core } & $\begin{array}{c}\mathrm{dI}_{0} / \mathrm{d} \ell \\
\left(\mathrm{mm}^{-1}\right)\end{array}$ \\
\hline GS1 & 2.1 \\
SCSN-81T(0.50) & 1.8 \\
PMP-doped polystyrene & 1.8 \\
\hline
\end{tabular}




\section{Figure captions}

Fig. 1 : Scintillating-fibre target oriented so as to provide event visualization in a plane transverse to the beam direction.

Fig. 2 : Arrangement of box number 1, containing active target, bent taper, and two proximity-focused image intensifiers (proxifiers). This part of the optoelectronic chain is designed to operate in regions of magnetic field, with the entrance plates of the proxifiers perpendicular to the direction of the magnetic field vector.

Fig. 3 : Arrangement of box number 2, containing an electrostatically focused image intensifier for magnification, a microchannel-plate image intensifier for strong light amplification, and a second electrostatically focused image intensifier for demagnification to match the size of the CCD.

Fig. 4 : Geometry of experimental set-up. A beam particle enters the target at a distance $z_{0}$ from the surface of detection. The beam line is at an angle $\theta$ with respect to the fibre axes.

Fig. 5 : Light patterns produced by $6 \mathrm{GeV} / \mathrm{c}$ hadrons crossing a target composed of fibres with a GS1 core, with target axis at $12^{\circ}$ with respect to the beam line. The scale is in millimetres at the target surface.

Fig. $6:$ Light patterns produced by $6 \mathrm{GeV} / \mathrm{c}$ hadrons crossing a target composed of fibres with an SCSN-81T $(0.50)$ core, with target axis at $20^{\circ}$ with respect to the beam line. The scale is in millimetres at the target surface.

Fig. 7 : Light patterns produced by $6 \mathrm{GeV} / \mathrm{c}$ hadrons crossing a target composed of fibres with a PMP-doped PS core, with target axis at $20^{\circ}$ with respect to the beam line. The scale is in millimetres at the target surface.

Fig. 8 : Spot profiles projected onto $\mathrm{x}$ and $\mathrm{y}$ axes (obtained for the glass target).

Fig. 9 : Distributions of track residuals.

Fig. 10 : Distributions of pulse height transverse to track direction as seen in two-dimensional projection at the target surface.

Fig. 11 : Attenuation in GS1 glass fibres. The light output is plotted as a function of the distance between the point of emission and the surface of detection, from track data recorded with the target at an angle of $12^{\circ}$ with respect to the beam line.

Fig. 12 : Attenuation in SCSN-81T(0.50) fibres. The mean total light output per track is plotted as a function of beam entry position along the target side, with the target at an angle of $20^{\circ}$ with respect to the beam line.

Fig. 13 : Attenuation in PMP fibres. The mean total light output per track is plotted as a function of beam entry position along the target side, with the target at an angle of $50^{\circ}$ with respect to the beam line.

Fig. 14 : Integrated light intensity at the MCP as a function of time, for emission commencing in the scintillator at time $t=0$.

Fig. 15 : Effect of concentration of WLS on light output in plastic fibres. The scintillation light is produced by $6 \mathrm{GeV} / \mathrm{c}$ hadrons traversing a composite target constructed from $1 \mathrm{~mm}$ diameter circular bundles of plastic fibres, having dimensions the same as those used for the standard targets [10]. From left to right in the figure, the beam particles passed through standard SCSN-81T fibres, SCSN-81T(0.50) fibres, and PMP-doped polystyrene fibres. A sample track is shown, with bundle outlines superimposed (top), and the light output (both track-associated and non-track-associated) is plotted across the target (bottom). 


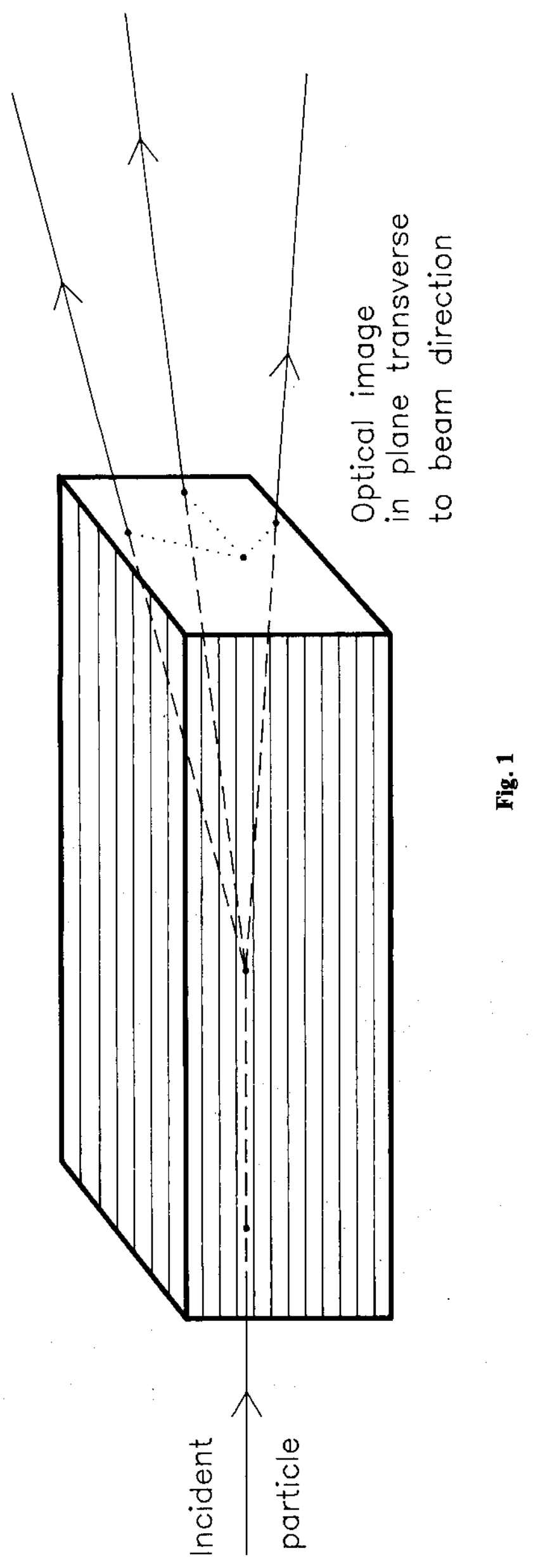




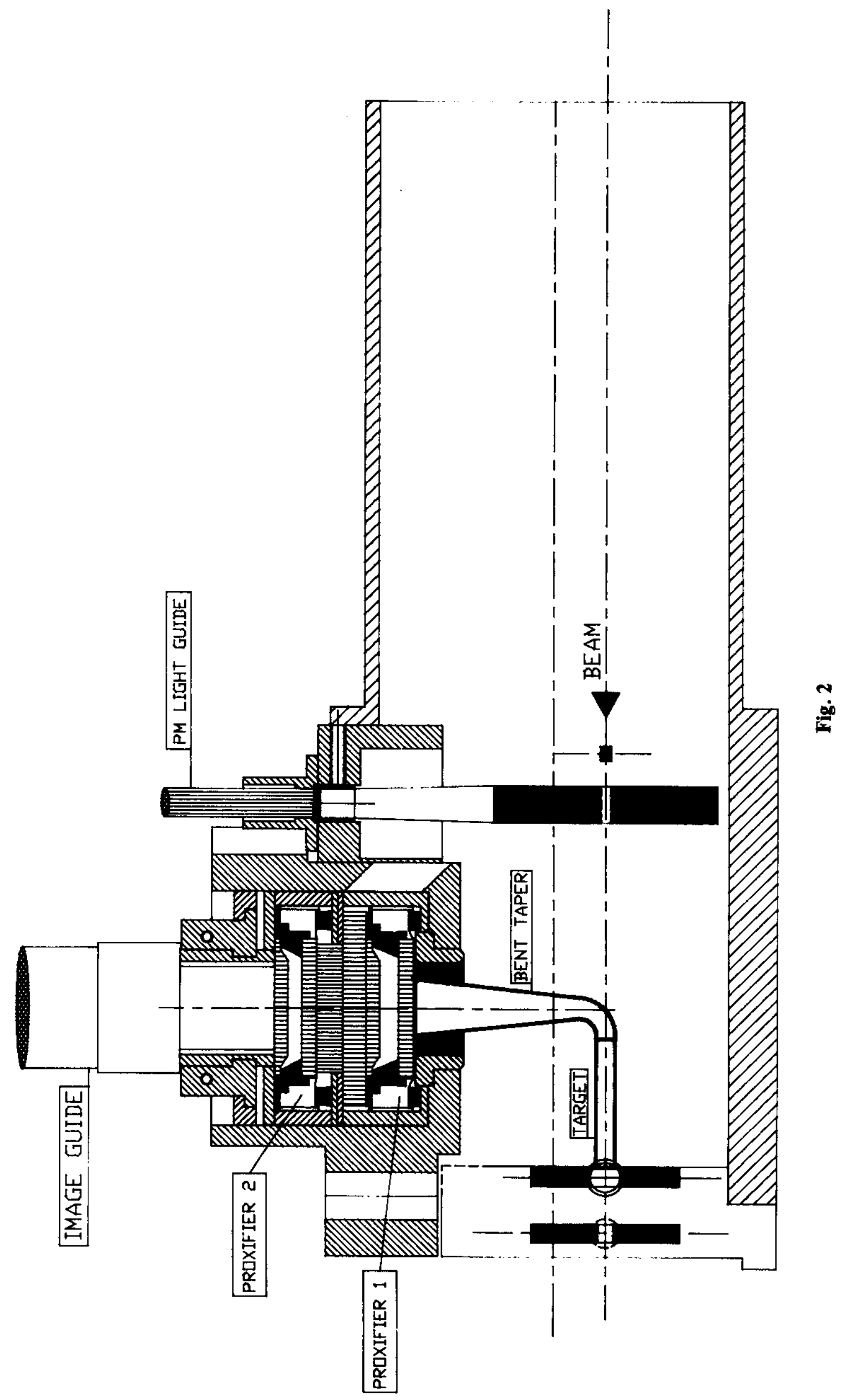




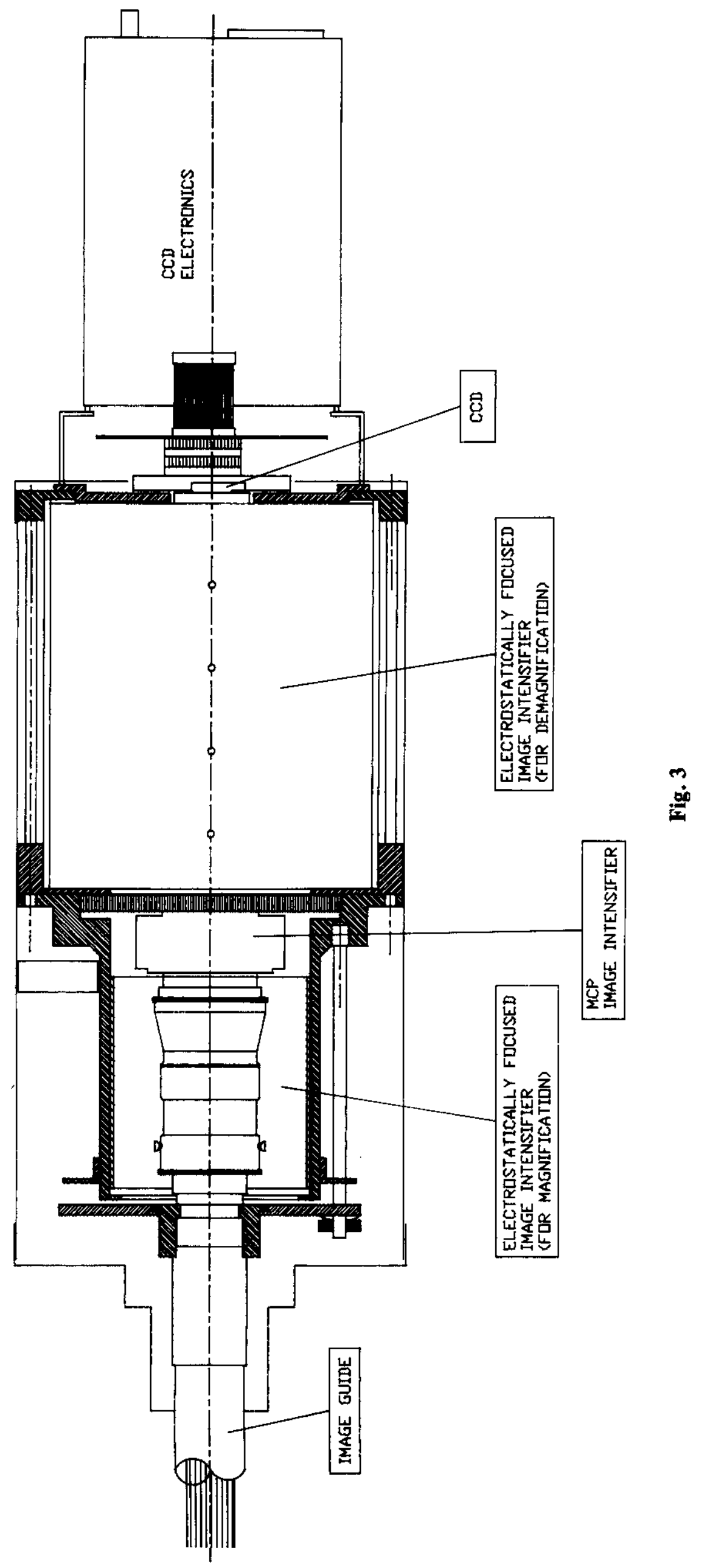




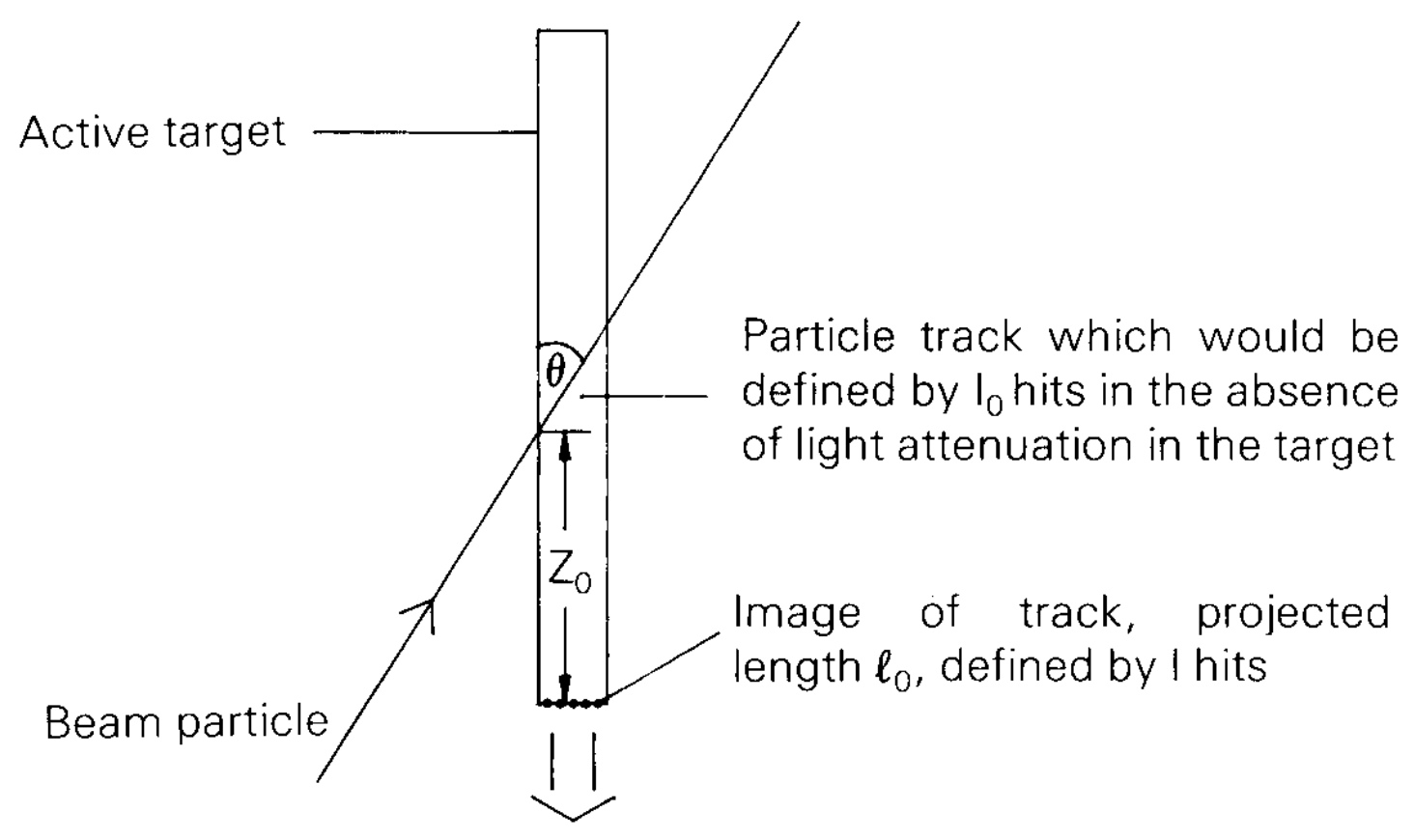

Optoelectronic chain

Fig. 4 

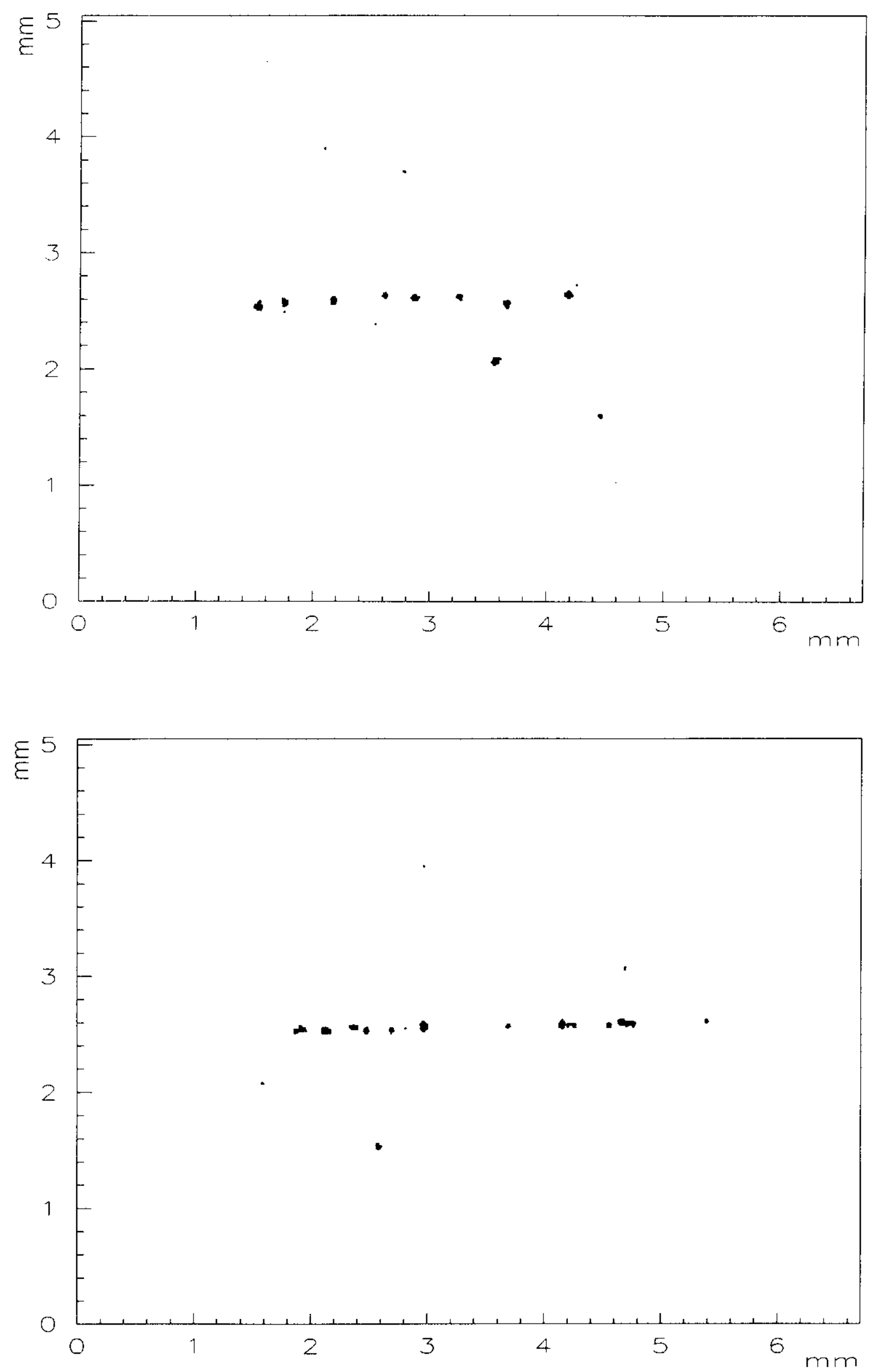

Fig. 5 

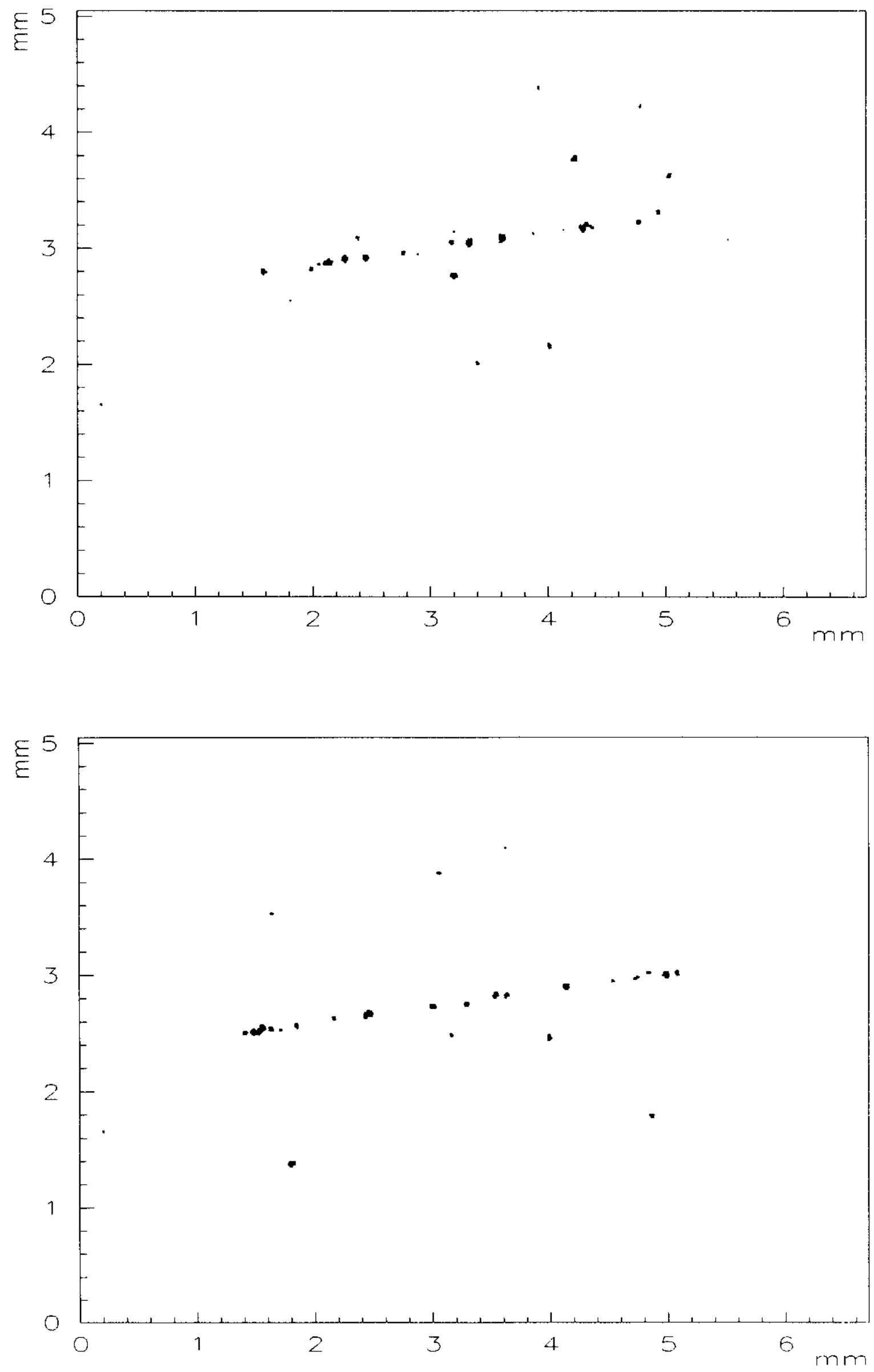

Fig. 6 

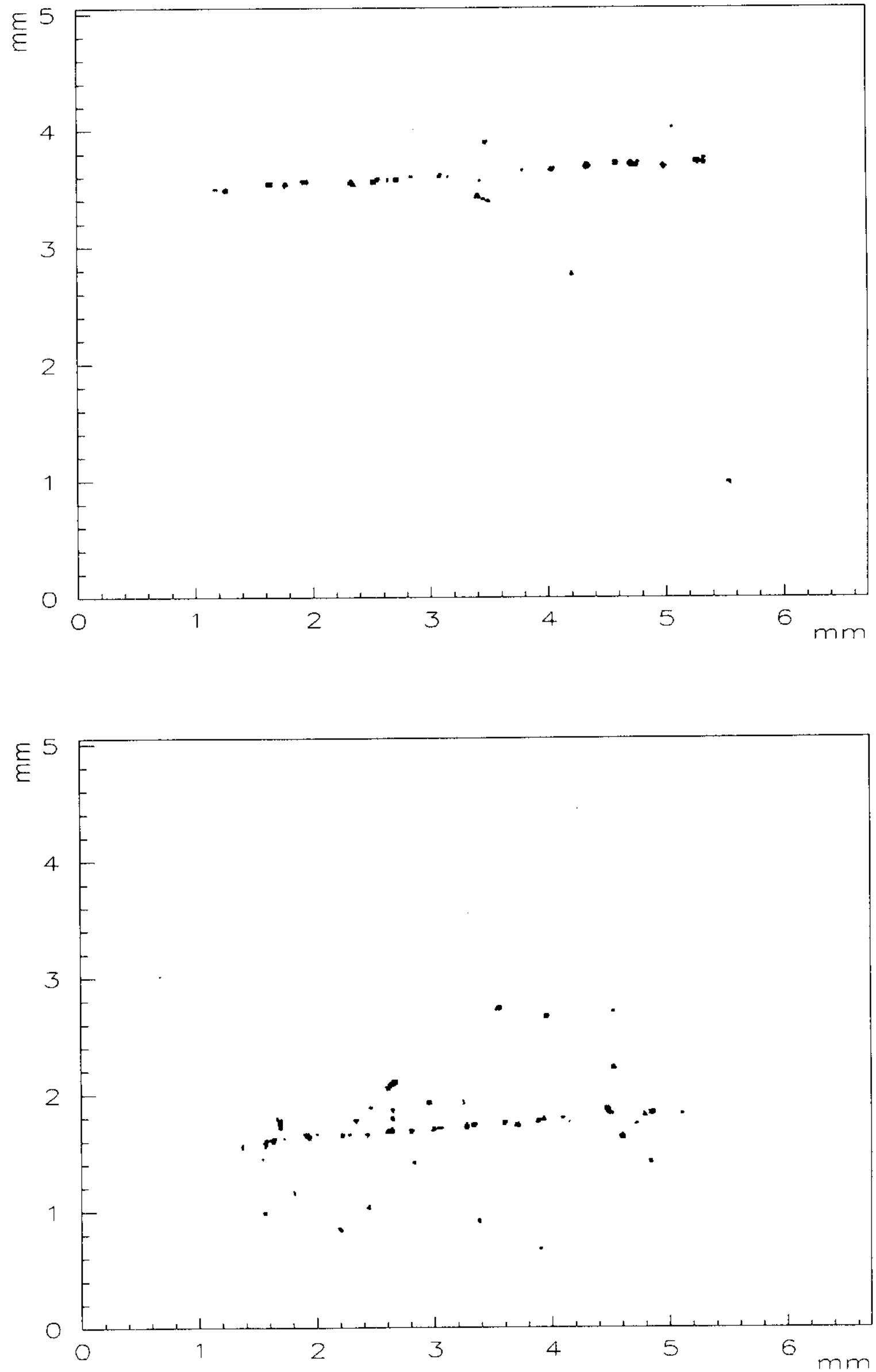

Fig. 7 

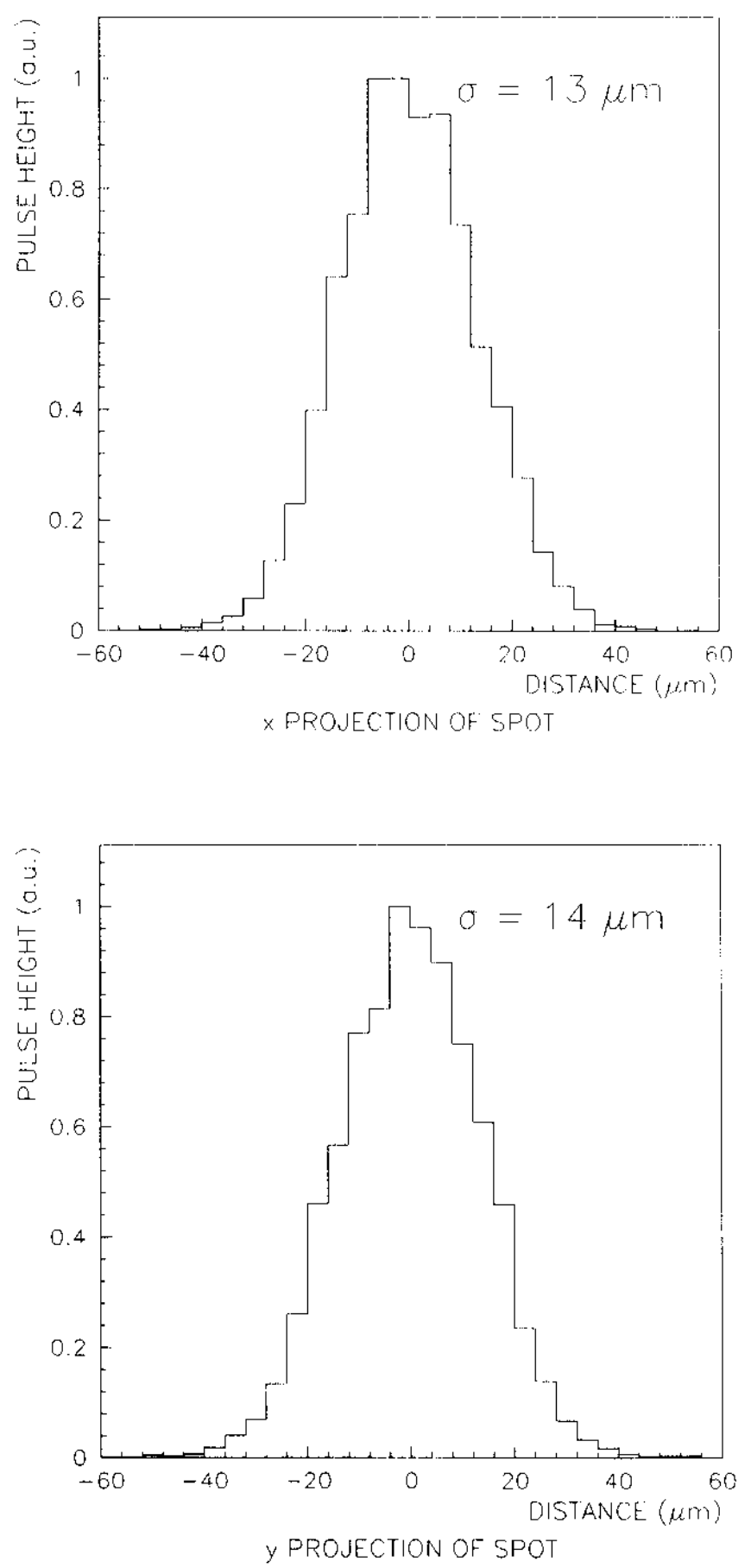

Fig. 8 

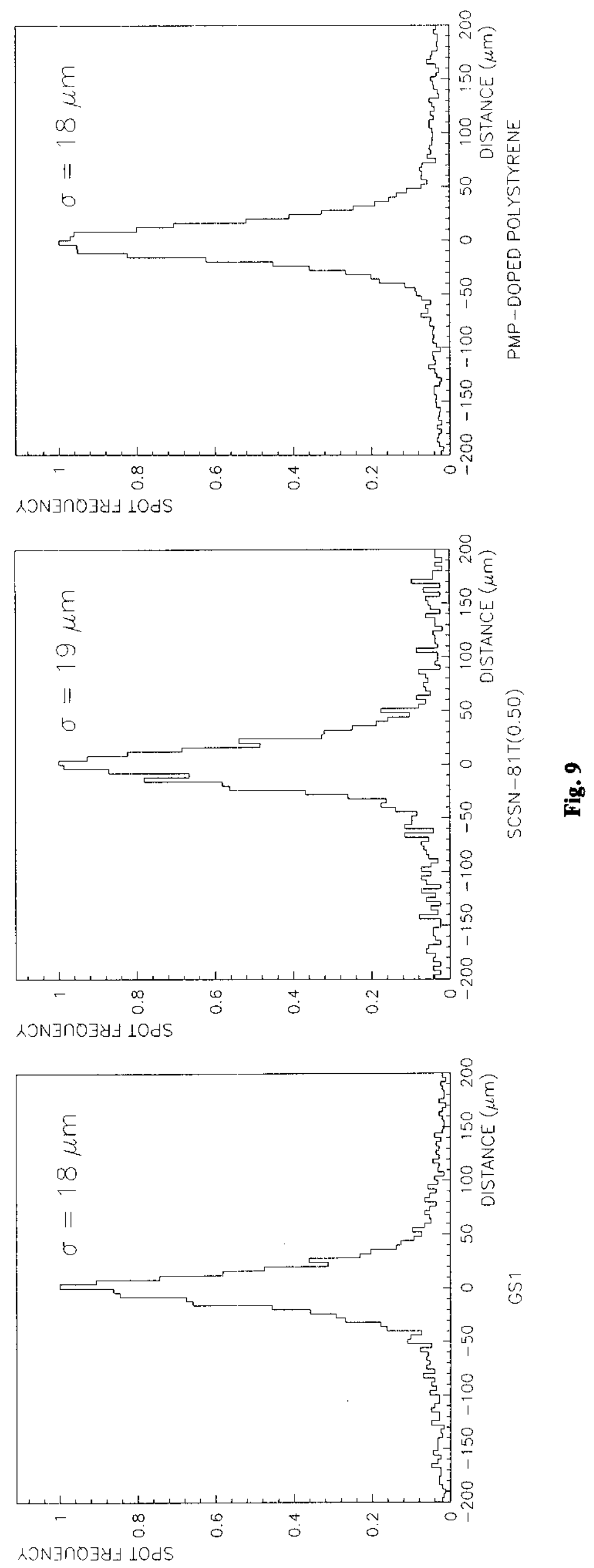

4 


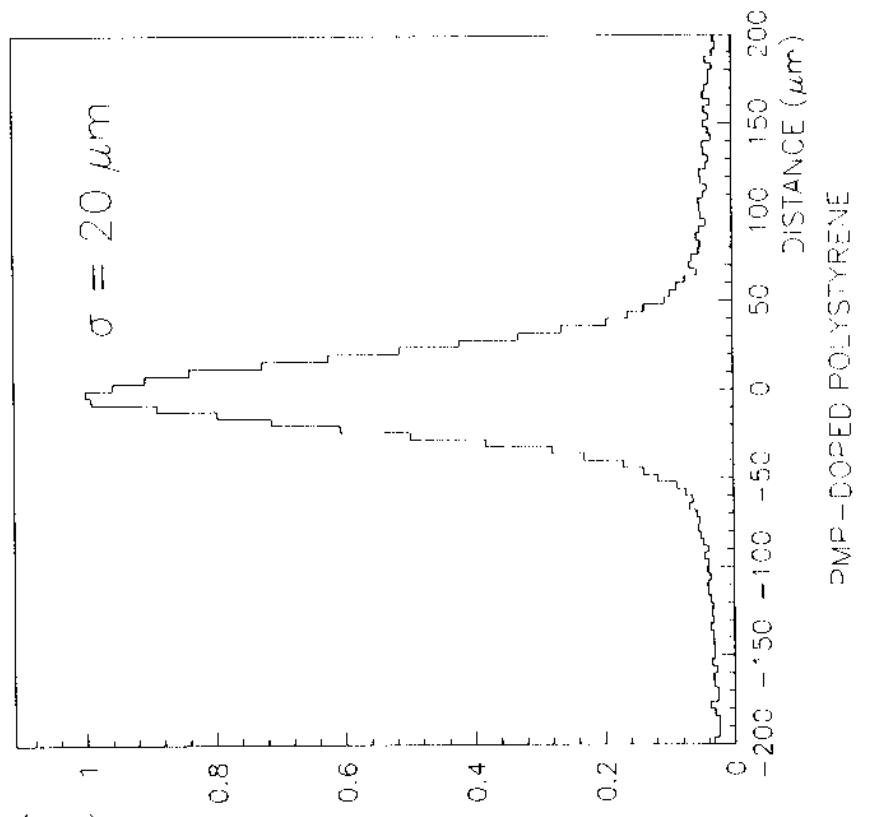

( n'D) $\perp$ HOI $\exists H \quad \exists S 7 n d$

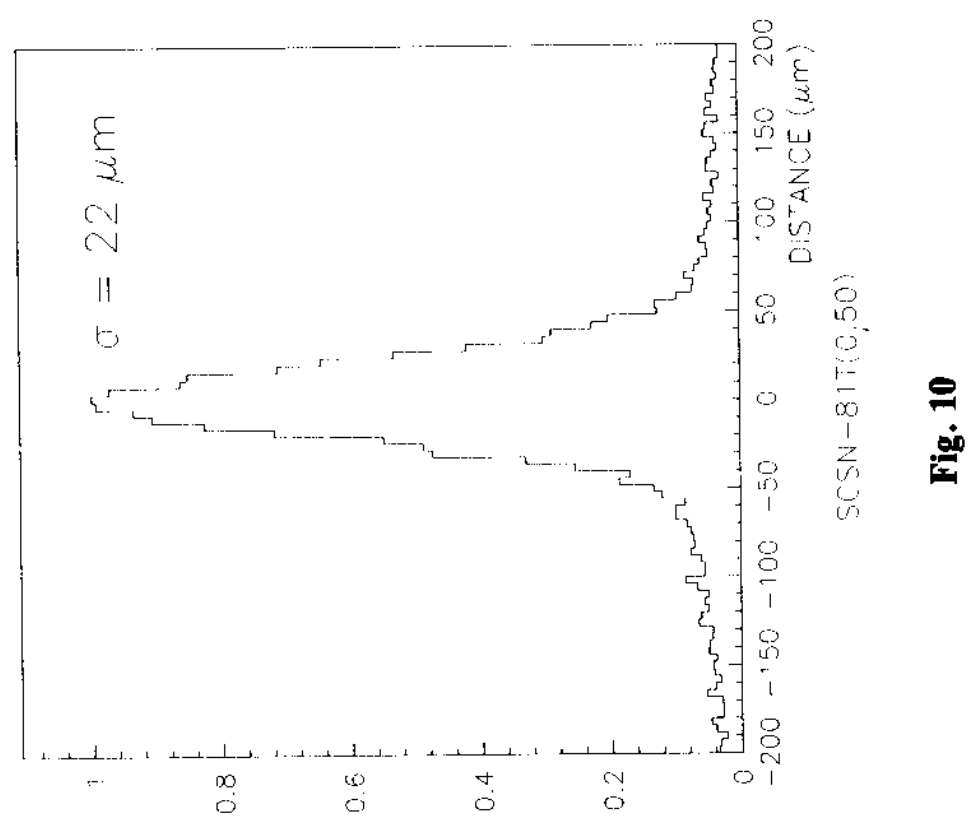

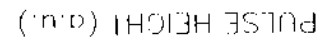

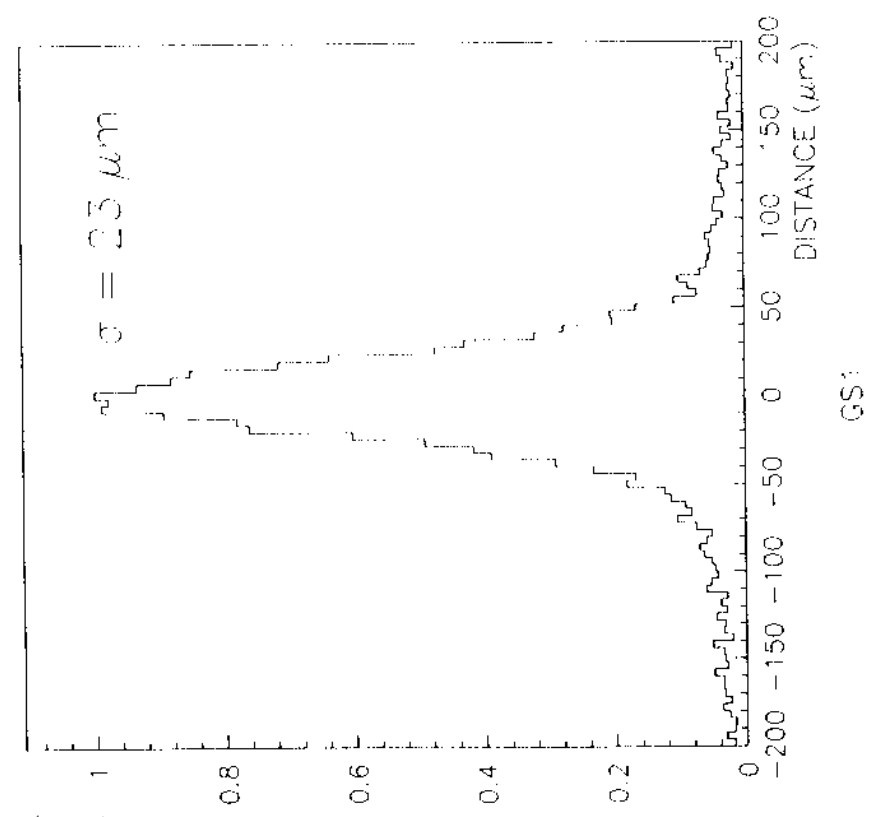

(.n.0) 1 HOIJH JSרnd 


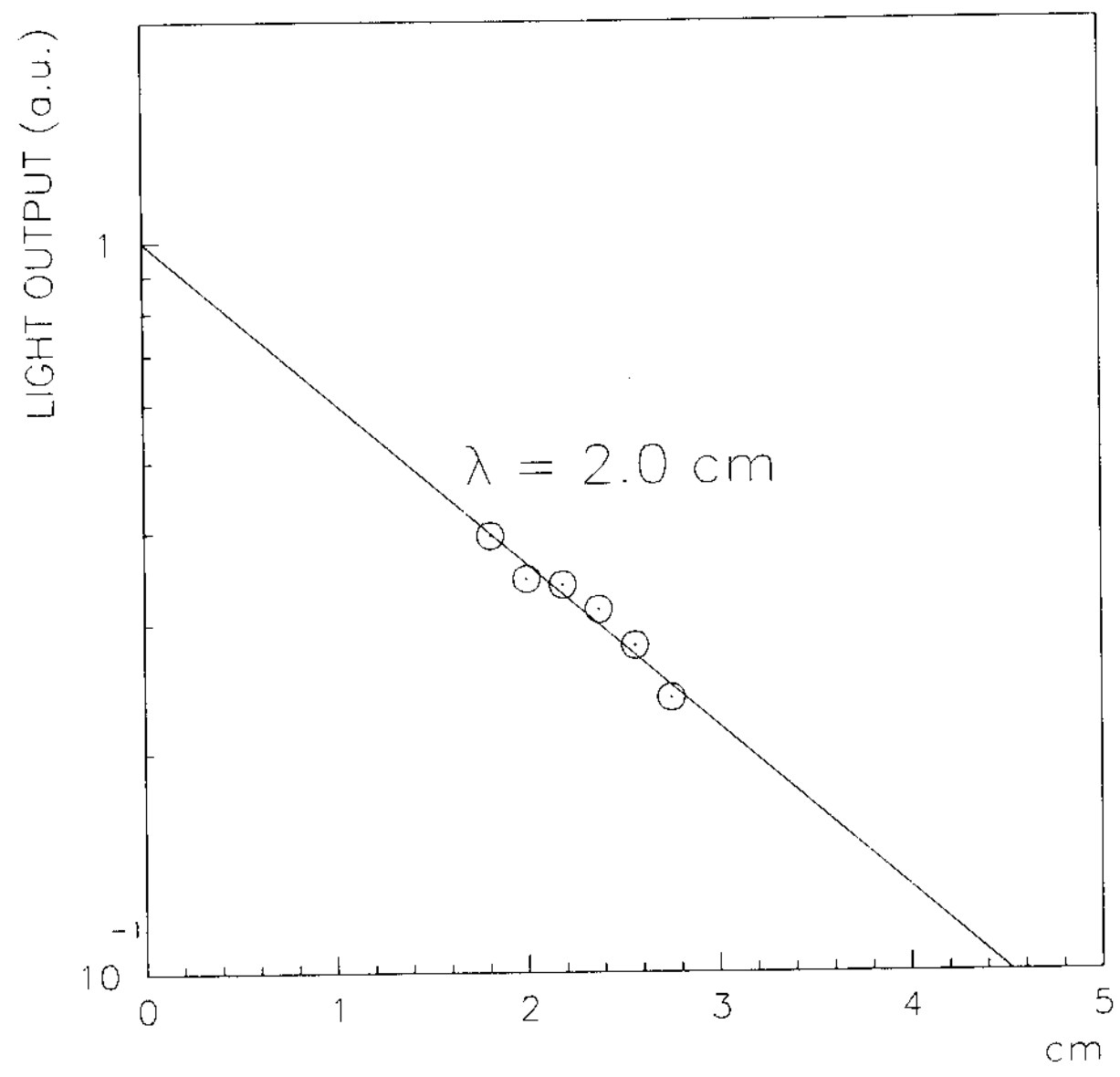

Fig. 11

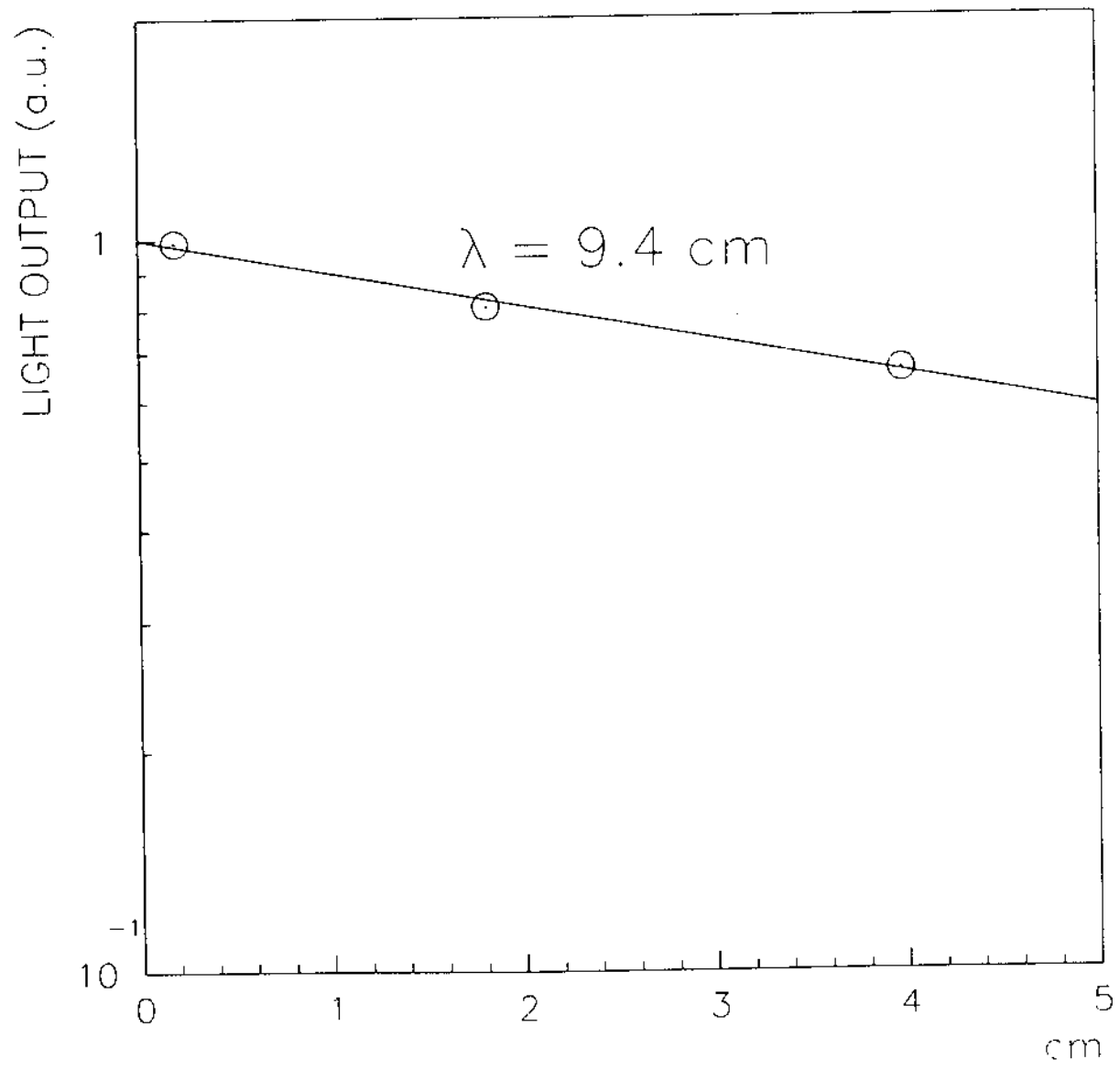

Fig. 12 


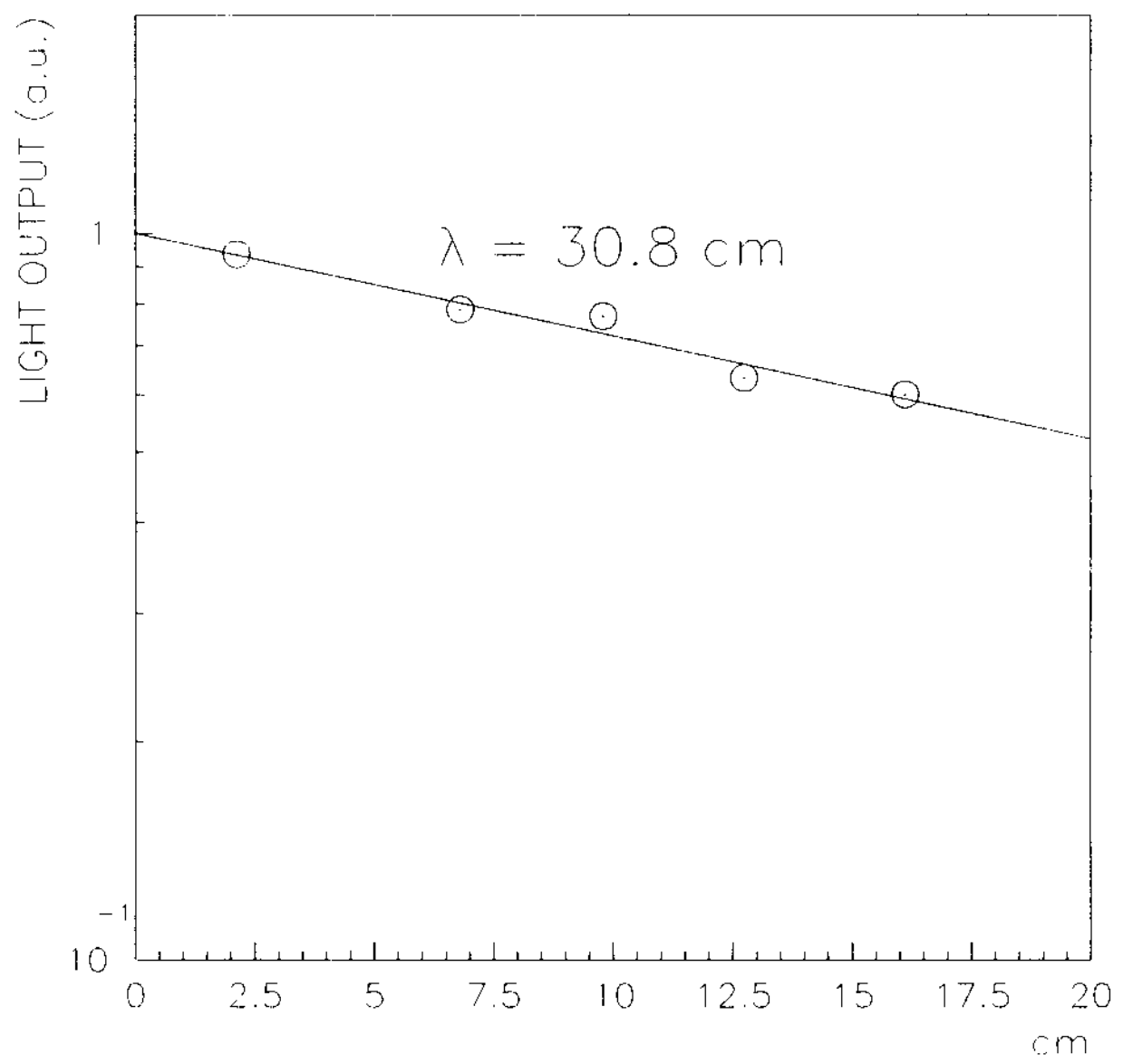

Fig. 13

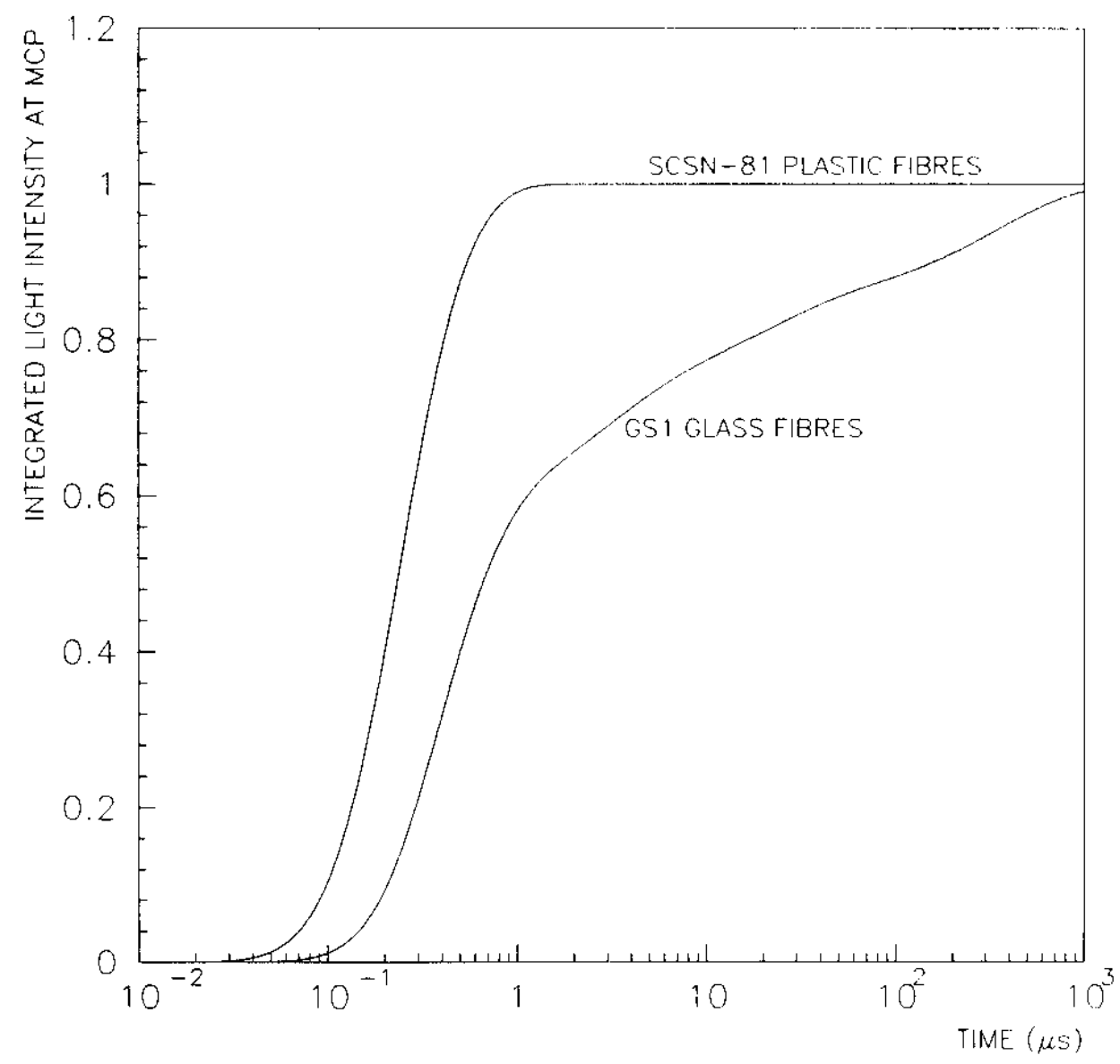

Fig. 14 

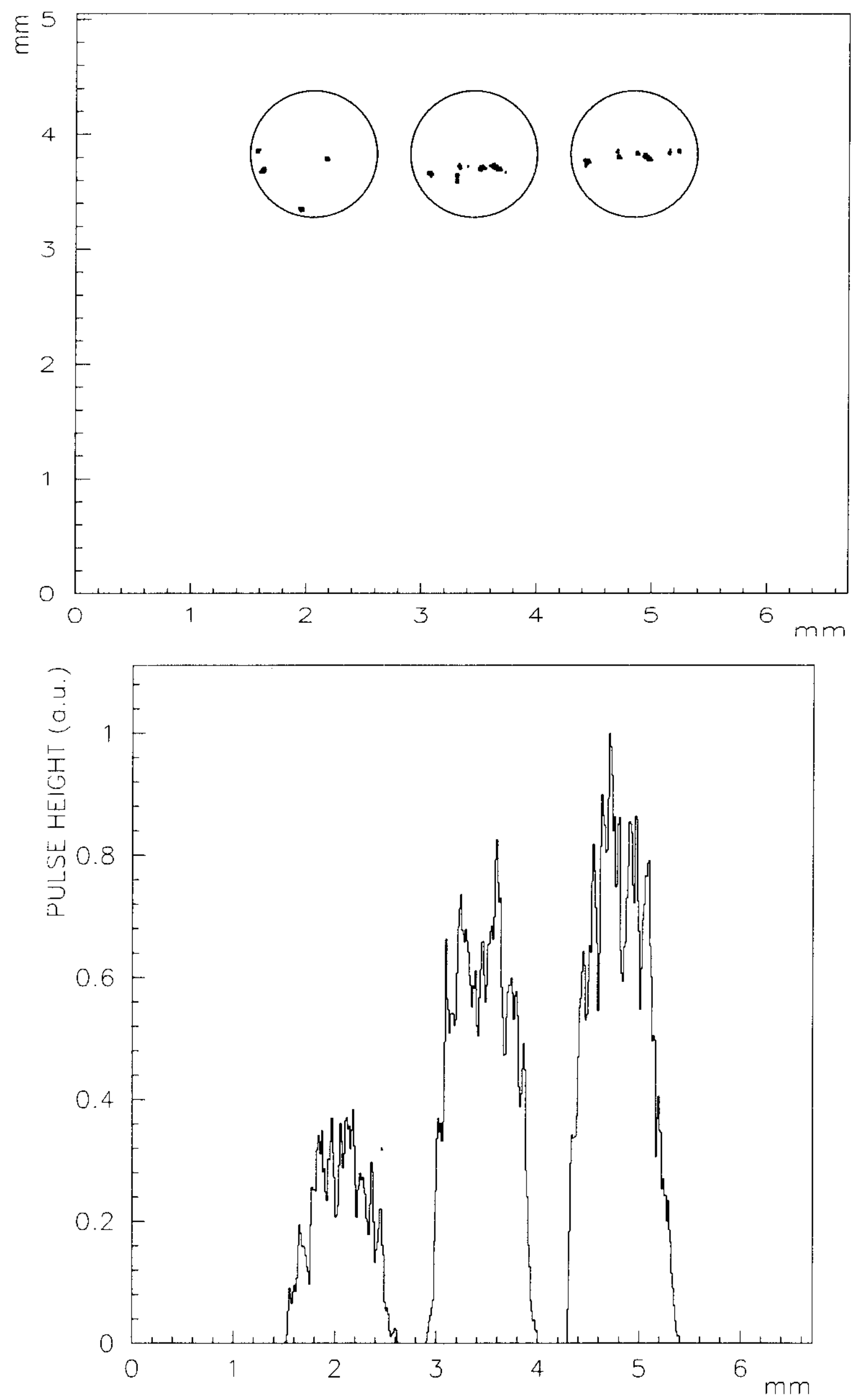

Fig. 15 